\title{
Proteasome inhibition and oxidative reactions disrupt cellular homeostasis during heme stress
}

\author{
F Vallelian ${ }^{1}$, JW Deuel ${ }^{1}$, L Opitz ${ }^{2}$, CA Schaer ${ }^{1,3}$, M Puglia ${ }^{1,2}$, M Lönn $^{4}$, W Engelsberger ${ }^{1}$, S Schauer ${ }^{2}$, E Karnaukhova ${ }^{5}$, DR Spahn ${ }^{3}$, \\ R Stocker ${ }^{4,6}$, PW Buehler ${ }^{5}$ and DJ Schaer ${ }^{*, 1,7}$
}

Dual control of cellular heme levels by extracellular scavenger proteins and degradation by heme oxygenases is essential in diseases associated with increased heme release. During severe hemolysis or rhabdomyolysis, uncontrolled heme exposure can cause acute kidney injury and endothelial cell damage. The toxicity of heme was primarily attributed to its pro-oxidant effects; however additional mechanisms of heme toxicity have not been studied systematically. In addition to redox reactivity, heme may adversely alter cellular functions by binding to essential proteins and impairing their function. We studied inducible heme oxygenase (Hmox1)-deficient mouse embryo fibroblast cell lines as a model to systematically explore adaptive and disruptive responses that were triggered by intracellular heme levels exceeding the homeostatic range. We extensively characterized the proteome phenotype of the cellular heme stress responses by quantitative mass spectrometry of stable isotope-labeled cells that covered more than $\mathbf{2 0 0 0}$ individual proteins. The most significant signals specific to heme toxicity were consistent with oxidative stress and impaired protein degradation by the proteasome. This ultimately led to an activation of the response to unfolded proteins. These observations were explained mechanistically by demonstrating binding of heme to the proteasome that was linked to impaired proteasome function. Oxidative heme reactions and proteasome inhibition could be differentiated as synergistic activities of the porphyrin. Based on the present data a novel model of cellular heme toxicity is proposed, whereby proteasome inhibition by heme sustains a cycle of oxidative stress, protein modification, accumulation of damaged proteins and cell death. Cell Death and Differentiation (2015) 22, 597-611; doi:10.1038/cdd.2014.154; published online 10 October 2014

Free heme can accumulate in hemolytic conditions during rhabdomyolysis and locally in wounded or inflamed tissues. ${ }^{1}$ The concentration of free heme in the extracellular space and within cells must be controlled within a narrow homeostatic range to avoid cytotoxicity and tissue damage caused by heme stress. ${ }^{2}$

Extracellular release from hemoproteins, cellular uptake, and intracellular metabolism determine the cumulative exposure of cells and tissues to heme. ${ }^{1}$ The hemoglobin $(\mathrm{Hb})$ and heme scavenger proteins haptoglobin and hemopexin restrict the accumulation of free heme within the extracellular space and prevent uncontrolled translocation into susceptible cells. ${ }^{3,4}$ Within cells, heme is continuously degraded by heme oxygenases $(\mathrm{Hmox}){ }^{5-8}$ The heme oxygenase system includes the constitutively expressed Hmox2 and inducible Hmox1 that is induced by acute increases in cellular heme such as during exogenous heme exposure. ${ }^{9}$ Cellular heme toxicity can result if excessive extracellular release exceeds the metabolic heme degradation capacity or if Hmox activity is inadequately low, such as that observed in rare conditions associated with loss-of-function mutations in the Hmox1 gene. $^{10}$

Several mechanisms of heme-triggered cell damage have been explored previously, with a focus on oxidative processes that can be catalyzed by free heme as well as on the activation of innate immunity receptors by the porphyrin. ${ }^{3,11-17}$ However, there is limited understanding of the 'metabolic' disruption that occurs in cells when intracellular free heme exceeds homeostatic levels and causes toxicity. To identify novel mechanisms of heme-triggered cell damage, we systematically explored heme-driven deviations of the cellular proteome phenotype and their underlying molecular mechanisms.

The primary signals that consistently appeared throughout our studies suggested that secondary to oxidative processes, the dysfunction of cellular protein homeostasis was the most important component of heme toxicity. These effects could be traced mechanistically to an inhibitory function of the porphyrin in the principal cellular protein degradation machinery: the proteasome.

\footnotetext{
${ }^{1}$ Division of Internal Medicine, University of Zurich, $\mathrm{CH}-8091$ Zurich, Switzerland; ${ }^{2}$ Functional Genomics Center Zurich, Swiss Federal Institute of Technology Zurich/ University of Zurich, Zurich, Switzerland; ${ }^{3}$ Institute of Anesthesiology, University of Zurich, Zurich, Switzerland; ${ }^{4}$ School of Medical Sciences, Discipline of Pathology and Bosch Institute, University of Sydney, Sydney, New South Wales, Australia; ${ }^{5}$ Laboratory of Biochemistry and Vascular Biology, Center of Biologics Evaluation and Research (CBER), FDA, Bethesda, MD, USA; ${ }^{6}$ Victor Chang Cardiac Research Institute and University of New South Wales, Sydney, New South Wales, Australia and ${ }^{7}$ Center of Evolutionary Medicine, University of Zurich, Zurich, Switzerland

*Corresponding author: DJ Schaer, Division of Internal Medicine, University Hospital Zurich, Ramistrasse 100, CH-8091 Zurich, Switzerland. Tel: +41 442552382 ; Fax +41 44255 4426; E-mail: dominik.schaer@usz.ch

Abbreviations: Hmox, heme oxygenase; Hb, hemoglobin; MEF, mouse embryo fibroblast; SILAC, stable isotope labeling by amino acids in cell culture; AMC, 7-amino-4methylcoumarin; CD, circular dichroism; SPR, surface plasmon resonance; CoPP, cobalt protoporphyrin; TBARS, thiobarbituric acid reactive substances; ACN, acetonitrile; AUC, area under the curve; GSH, glutathione; DTT, dithiothreitol; SRM, selected reaction monitoring; nanoLC - MSMS, liquid chromatography nanospray-tandem mass spectrometry

Received 07.4.14; revised 04.8.14; accepted 21.8.14; Edited by J Cidlowski; published online 10.10.14
} 


\section{Results}

A cell culture model of cellular heme toxicity. We have developed a cell culture model to study changes in protein expression that were specifically triggered by intracellular heme accumulation. We generated mouse embryo fibroblast (MEF) cell lines from wild-type and Hmox1 knockout mice (Figure 1). With this model, intracellular heme levels and resulting heme toxicity could be experimentally controlled by extracellular heme exposure of cells with high and low intracellular heme degradation capacity, respectively.

The Hmox1 (-/-) MEF cells expressed no functional Hmox1 mRNA (Figure 1a) and as a result accumulated more cell-associated heme during extracellular exposure compared with wild-type cells (Figure 1b). During heme exposure the Hmox1 ( $-/-)$ cells show a dose-dependent decrease in mitochondrial function as indicated by decreased cellular ATP (Figure 1c) and parallel induction of caspase 3/7 activity (Figure 1d) as well as nuclear condensation (Figure 1e) that occurred at heme concentrations exceeding $10 \mu \mathrm{M}$. In contrast, Hmox1-expressing MEFs were resistant to heme exposure up to an extracellular concentration of $40 \mu \mathrm{M}$. Addition of the high-affinity heme scavenger hemopexin, which prevents translocation of heme across the cell membrane, completely prevented the toxic heme response in Hmox1 $(-/-)$ MEFs that was characterized morphologically by nuclear condensation and loss of regular cytoskeleton (Figure 1e). Collectively, these data indicate that this cell culture model allows for a systematic evaluation of cellular responses that are specifically triggered by levels of intracellular heme exceeding the homeostatic range.

Heme exposure induces a proteome signature of impaired protein degradation and oxidative stress. To systematically study heme-triggered cellular responses, we explored the proteome phenotype during different heme exposure conditions by mass spectrometry in combination with stable isotope labeling by amino acids in cell culture (SILAC). ${ }^{18}$ Differentially expressed proteins were identified in two independent Hmox1 $(+/+)$ cells and in two Hmox1 $(-/-)$ cell lines that were treated with heme at 5 or $10 \mu \mathrm{M}$ for a period of 12 or $24 \mathrm{~h}$, respectively. For the quantitative analysis we considered all proteins that were identified by at least two peptides in at least two experiments per condition. Across all experiments, 2068 proteins fulfilled these criteria. The protein expression data of the two Hmox $1(+/+)$ cell lines were pooled for further quantitative analysis, because both cell lines showed only minimal and very similar heme-triggered responses. Figure $2 \mathrm{a}$ shows the number of experiments and the fraction of differentially expressed proteins (candidates) for each treatment condition and cell type, respectively, and indicates that more extensive proteome changes occurred in heme-treated Hmox $1(-/-)$ cells compared with heme-treated Hmox $(+/+)$ cells. Furthermore, a correlation analysis of the significantly regulated proteins across all experimental conditions identified a clear genotype discriminating clustering of the protein expression data (Figure $2 b$ ). This indicates that the control of intracellular heme by Hmox1 critically determines the cellular response to heme exposure in our model. Figure 2c shows a hierarchical clustering analysis of all SILAC experiments and includes all proteins for which significant differential protein expression of $>1.4$-fold (treated versus control; $P<0.05$ ) was found in at least one condition. Heavy and light chains of ferritin and Hmox1 represent the strongly induced common protein expression signature across all experiments. In the knockout cells, Hmox1 represents a small nonfunctional protein remnant that can still be detected by mass spectrometry. Beyond this common protein expression signature, the toxic heme response of Hmox1 (-/-) MEF cells was uniquely characterized by a cluster of strongly upregulated proteins. Within this cluster we identified ubiquitin, the ubiquitin adaptor protein sequestosome/p-62 (Sqstm1), a number of heat shock proteins, as well as antioxidant defense proteins (i.e., peroxiredoxins, glutathione-S-transferase, and thioredoxin reductase). Importantly, many of these proteins such as Sqstm1 were also upregulated in Hmox1 $(+/+)$ cells but to a much lower level than in heme-stimulated Hmox1 (-/-) cells, indicating that the level of intracellular heme is the critical response determinant. Collectively, our data identified a specific set of signature proteins that characterizes the adaptive and disruptive metabolic changes that occur as a response to cellular heme stress. Detailed mass spectrometry and protein expression data can be found at http://fgczgstore.uzh.ch/public/p901/.

We analyzed the heme-upregulated protein cluster for enriched protein networks by Metacore (online version, Thomson Reuters; https://portal.genego.com). The most significantly enriched networks were related to the response to unfolded proteins $\left(F D R=6.1 \times 10^{-13}\right)$, general protein folding, as well as the response to oxidative stress $\left(\mathrm{FDR}=1.4 \times 10^{-5}\right)$. This suggests that excessive intracellular heme might not only act as an oxidizing agent but also disrupt critical protein homeostasis pathways. Controlled protein degradation is critical for a cell to recover from the oxidative damage that is the primary impact expected to result from high-level heme exposure. Blocking of this essential repair mechanism may exacerbate heme toxicity. The accumulation of ubiquitin and the ubiquitin adaptor Sqstm1 provide the most direct evidence for impaired protein degradation capacity in heme-stressed cells. We confirmed by selected reaction monitoring (SRM) mode mass spectrometry (Figure 3a) and western blot (Figures 3c and d), respectively, that heme increased Sqstm1, ubiquitin, and Hsp70, with a much stronger response in Hmox1 (-/ - ) compared with Hmox1 (+/+) MEF cells. We also confirmed ferritin light chain enhancement as a common response to heme exposure of Hmox1 (+/+) and Hmox1 $(-/-)$ MEF cells (Figure 3a).

To explore how transcriptional and post-transcriptional regulatory mechanisms contributed to these observations, we measured heme-induced mRNA expression of Sqstm1 and ubiquitin (Ubc) by RT-PCR as well as heme-triggered ubiquitin accumulation when protein synthesis was blocked by cycloheximide in Hmox1 $(-/-)$ MEF cells. These results suggested that post-transcriptional protein accumulation contributed in addition to transcriptional gene regulation to our observations. Both genes show heme-stimulated mRNA expression in Hmox1 ( $-/-)$ MEFs (Figure 3e). Inhibition of protein synthesis by cycloheximide $(100 \mu \mathrm{M})$ reduced baseline 

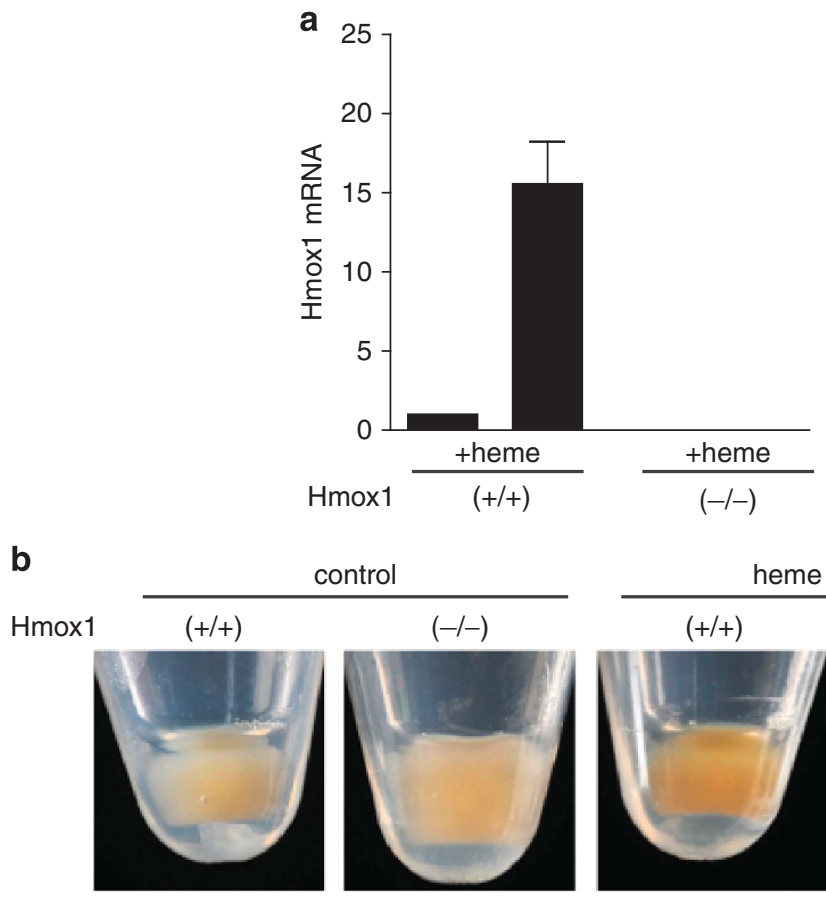

control
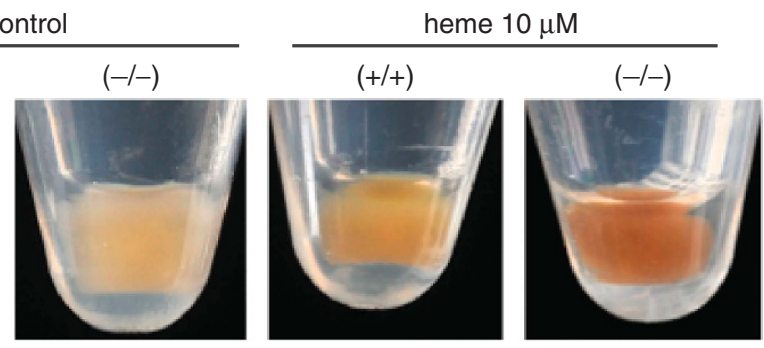

C
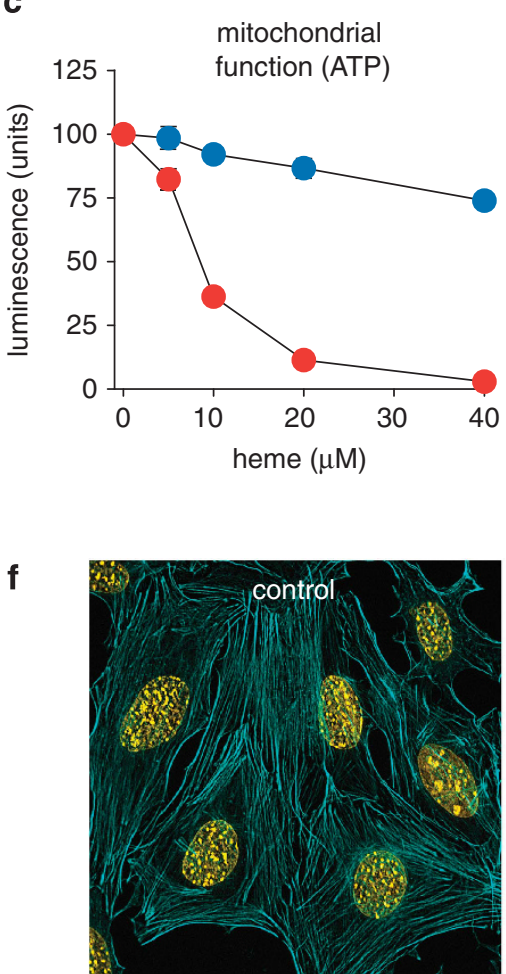

d

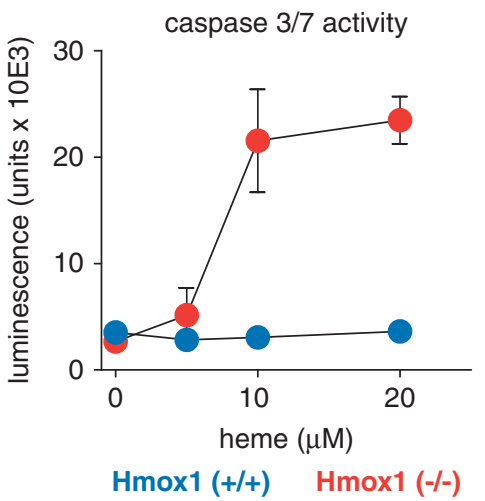

e

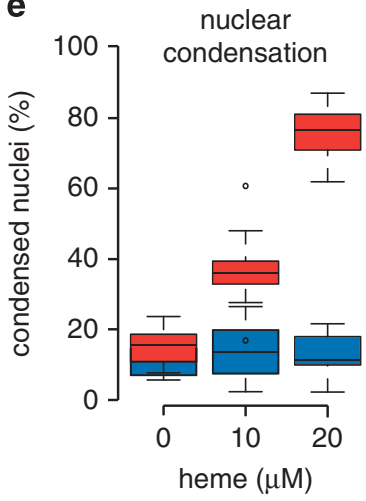

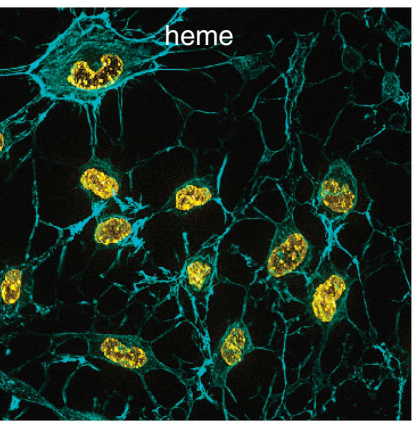

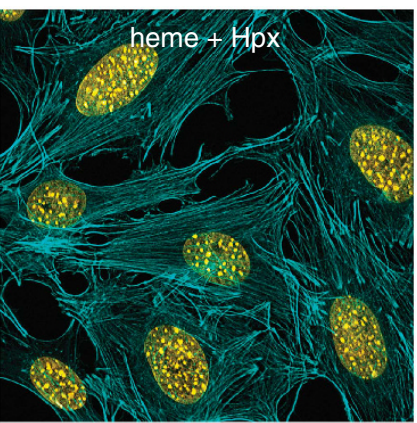

Figure 1 Cell culture model of heme toxicity. (a) Hmox1 $(+/+)$ and $\operatorname{Hmox} 1(-/-)$ cells were incubated with $10 \mu \mathrm{M}$ heme for $4 \mathrm{~h}$. The relative Hmox1 mRNA induction in response to heme exposure was quantified by TaqMan RT-PCR. The mRNA levels are indicated as fold expression over control. No transcript was detected in the Hmox 1 ( - I - ) cell line. (b) Cell pellets of Hmox1 $(+/+)$ and Hmox1 $(-/-)$ cells after $24 \mathrm{~h}$ of heme exposure $(10 \mu \mathrm{M})$. Before imaging, cells were washed three times in large volumes of PBS containing EDTA. (c and d) Hmox1 (+/+) MEFs (blue) and Hmox1 ( - / - ) MEFs (red) were treated with the indicated concentrations of heme for $12 \mathrm{~h}$. Intracellular ATP (c) and caspase 3/7 activity (d) were measured by luminescence assays. Data represent the mean \pm S.D. of six biologic replicates. (e) Hmox $1(+/+)$ MEFs (blue) and Hmox $1(-/-$ ) MEFs (red) were treated with the indicated concentrations of heme for $14 \mathrm{~h}$. Condensed nuclei were quantified by digital image analysis after Hoechst staining. Data include images (15 per replicate) from three independent biologic replicates. (f) Hmox $1(-/-)$ MEFs were incubated with or without $20 \mu \mathrm{M}$ heme for $12 \mathrm{~h}$ and stained with Hoechst (nuclei = yellow) and Alexa 488 phalloidin (actin cytoskeleton = cyan). The heme toxicity, which was characterized by cytoskeleton rarefication and nuclear condensation, was totally prevented by hemopexin $(\mathrm{Hpx} ; 0.5 \mathrm{mg} / \mathrm{ml})$. Images were acquired with a Zeiss Observer Z1 equipped with an ApoTome.2 module and an Axiocam MRm at $\times 400$ original magnification. ${ }^{\circ}$ indicates outlier values 
a

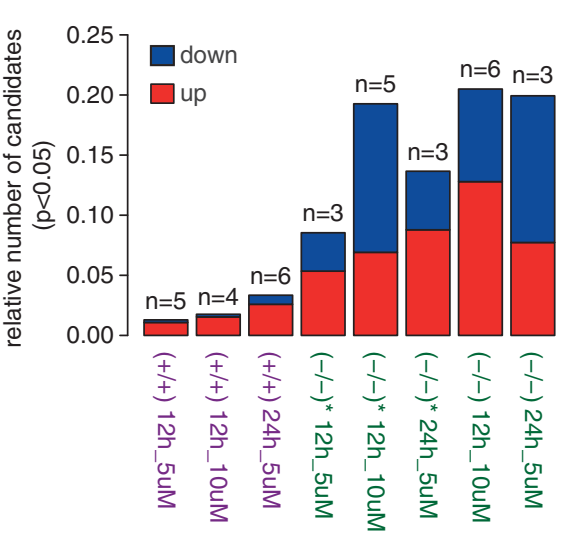

b

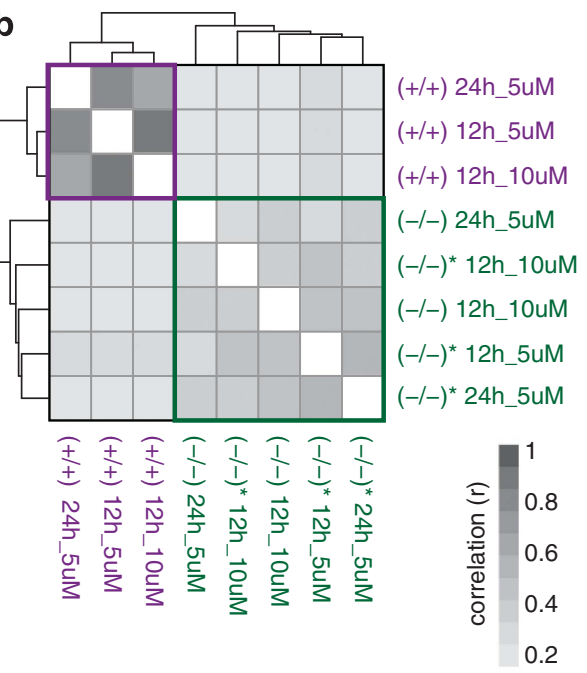

c

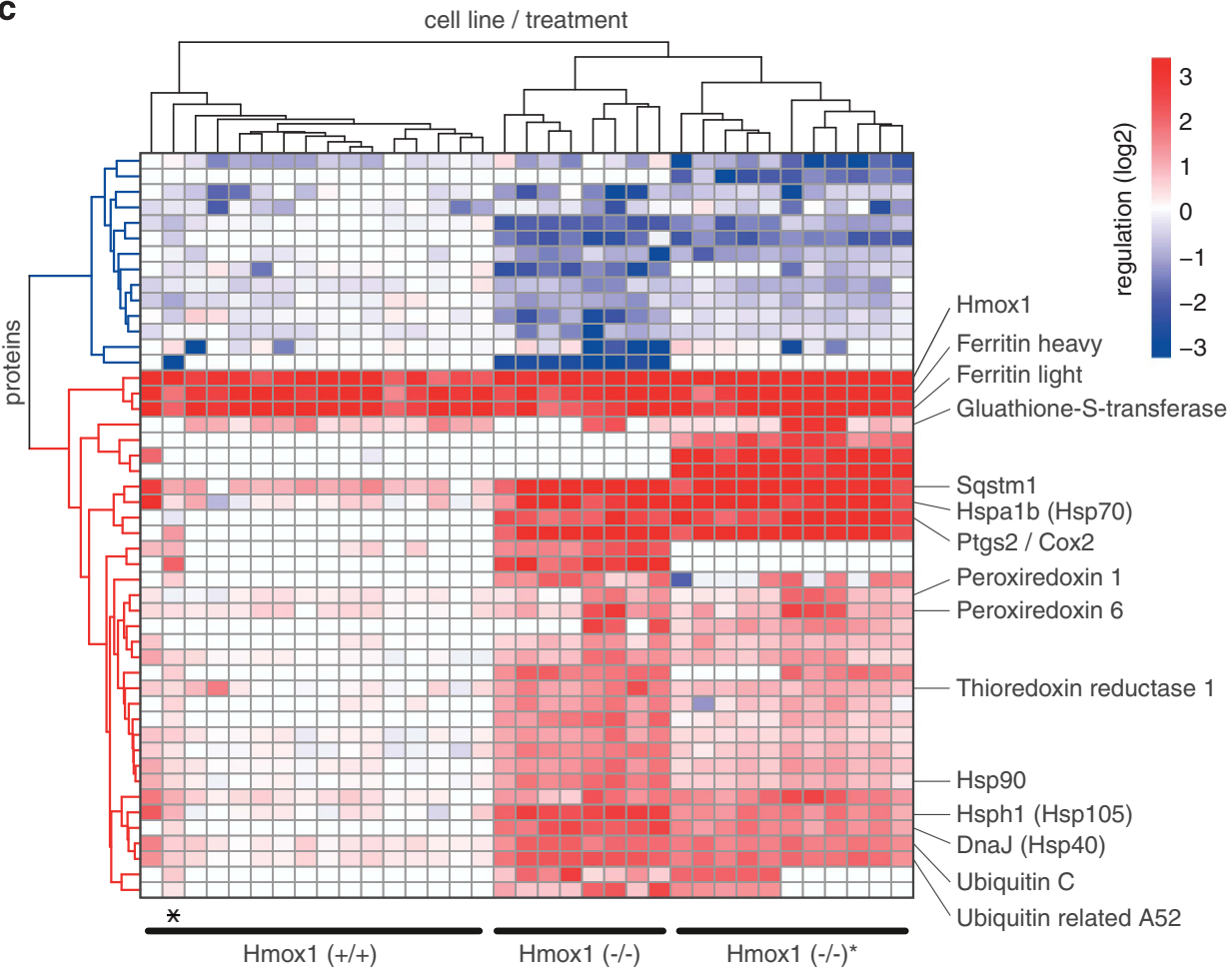

Figure 2 Proteome changes in response to uncontrolled cellular heme overload. (a) Number of experiments and fraction of proteins that were significantly changed compared with matched untreated cells $(P<0.05)$ for each treatment $x$ cell type condition. Hmox $(-/-)$ and $H \operatorname{mox}(-/-)^{*}$ are two independent cell lines derived from two independent mouse embryos. Red indicates fraction of upregulated proteins, and blue indicates fraction of downregulated proteins. (b) Correlation plot of the log2 expression ratios of all significantly changed proteins for each treatment $x$ cell type condition (darker $=$ stronger correlation of protein expression pattern between two conditions). The plot defines a clear segregation of the protein expression data according to Hmox1 expression status. (c) Hierarchical clustering analysis of all proteins that have been identified as differentially expressed in any of the tested conditions. Each column in the plot represents data from an individual SILAC experiment. The plot includes all proteins with an absolute expression ratio of $>0.5(\log 2)$ in treated compared with matched untreated control cells $(P<0.05)$. Red indicates protein expression is higher in treated cells, and blue indicates protein expression is lower in treated cells. The asterisk indicates a single $H \operatorname{mox}(-/-)$ experiment that clustered within the group of Hmox $(+/+)$ experiments. The identical plot with labeling information of all proteins can be found in Supplementary Figure 1

levels of ubiquitin-conjugated proteins as measured by western blot. However, cycloheximide did not prevent the appearance of large molecular ubiquitin-conjugated proteins in heme-treated Hmox1 (-/ - ) MEF cells (Figure 3f), suggesting that post-transcriptional processes contribute to the pattern of protein expresssion.
To further support that post-transcriptional mechanisms contribute to Sqstm1 accumulation in heme-treated cells, we expressed a myc-tagged Sqstm1 under the control of the constitutively active CMV promoter in RAW-264 cells. At higher extracellular heme concentrations, heme exposure dose dependently caused accumulation of myc-tagged 
a
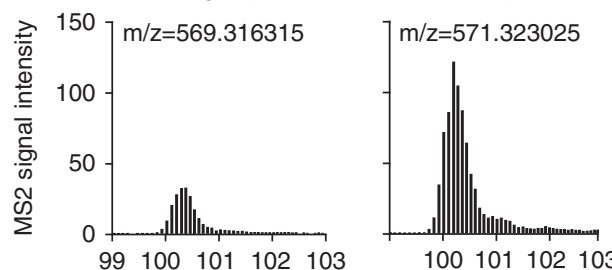

100101102103

Sqstm1 (AYLLGKEEATR)
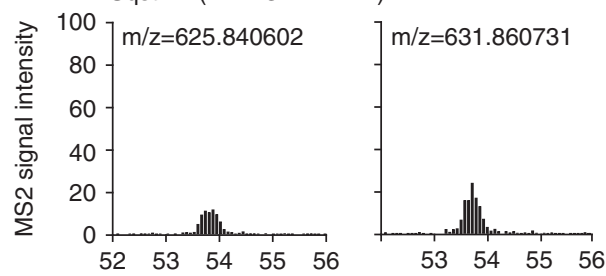

Ubiquitin (TITLEVEPSDTIENVK)
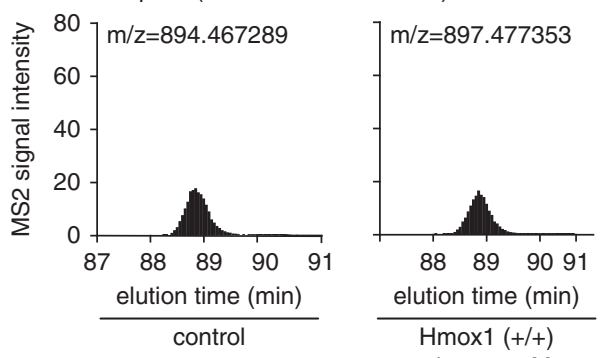

$\begin{array}{lll}88 & 89 & 9091\end{array}$

elution time $(\min )$

Hmox1 $(+/+)$
theme $5 \mu \mathrm{M}$

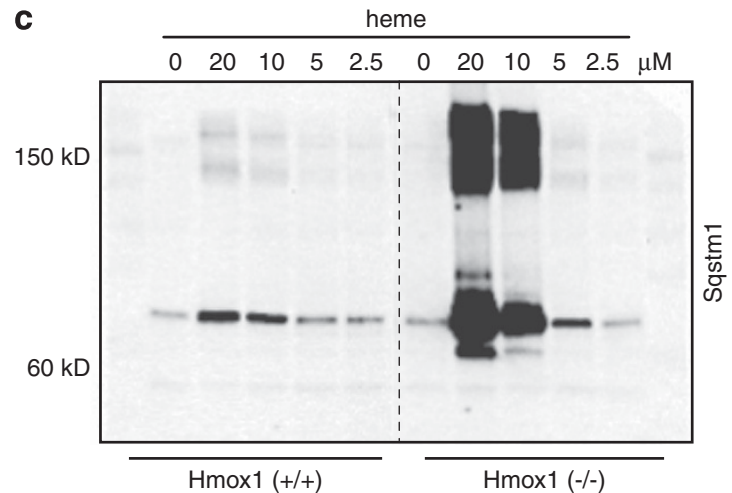

f

$\begin{array}{lllllllll}0 & 5 & 10 & 20 & 0 & 5 & 10 & 20 & \text { heme }\end{array}$

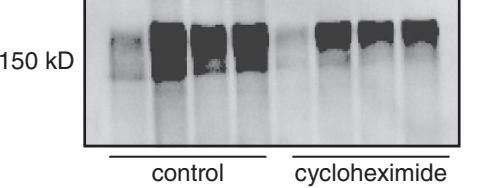

b

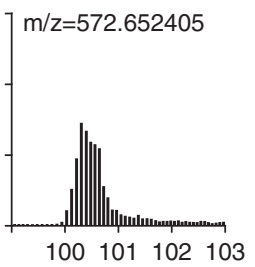

100101102103
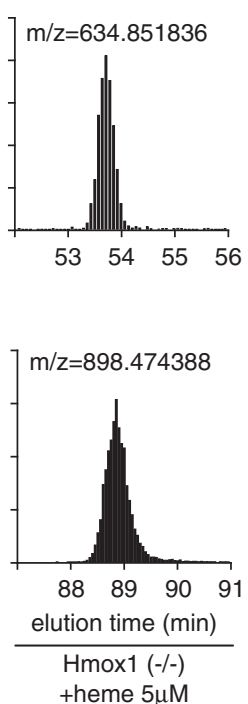

d

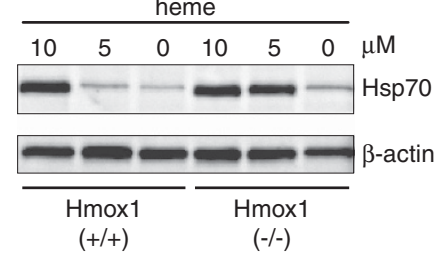

e 25

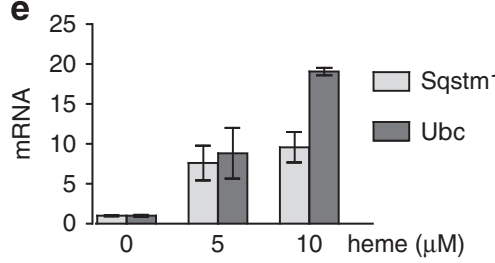

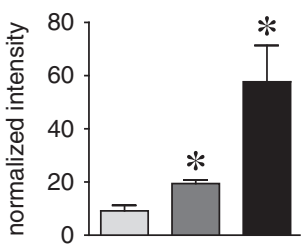

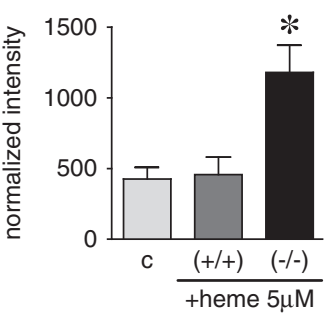

Figure 3 Expression of heme-triggered proteins. (a) Selected reaction mode (SRM) quantitative mass spectrometry of the ferritin light chain, Sqstm1, and ubiquitin. The graphs show elution profiles of the sum intensities of three SRM transitions of the indicated peptides. The $m / z$ indicates the precursor mass of the respective peptide from light, medium, and heavy labeled cells. (b) The bar graphs show normalized sum intensities of four peptides per protein \pm S.E.M. from three independent experiments $\left({ }^{\star} P<0.05\right.$ compared with control). (c and d) Detection of Sqstm1/p62 (c) and HSP-70 (d) by western blot of cell lysates from Hmox1 (+/+) and Hmox1 ( $-/-)$ MEF cells stimulated with a range of heme concentrations for $8 \mathrm{~h}$. (e) RT-PCR of Sqstm1 and Ubc in control and heme-stimulated (5h) Hmox1 ( $-/-$ ) MEFs. Relative mRNA quantities were normalized to Hprt expression. (f) Western blot (nonreducing) of ubiquitin in control and heme-stimulated (6h) Hmox1 ( $/-)$ MEFs. Incubations were in the presence or absence of cycloheximide $(100 \mu \mathrm{M})$ to inhibit de novo protein translation. (g) Anti-myc western blot of Sqstm1-myc expressed under a constitutive CMV promoter in stably transfected RAW-264 cells exposed to a range of heme concentrations 
Sqstm1 (Figure 3g). This result strongly supports that high cellular heme concentration impairs degradation of Sqstm1 by the homeostatic protein degradation pathways. These data, in addition to older biochemical studies that empirically used heme to inhibit proteasome function in biochemical assays, led us to the hypothesis that excessive intracellular heme could disrupt cellular protein homeostasis by an inhibitory action on the proteasome. ${ }^{19-23}$

Heme inhibits the ubiquitin-proteasome pathway and reduces $26 \mathrm{~S}$ proteasome activity. Figure $4 \mathrm{a}$ shows that during heme exposure, Sqstm1 accumulated in the

a
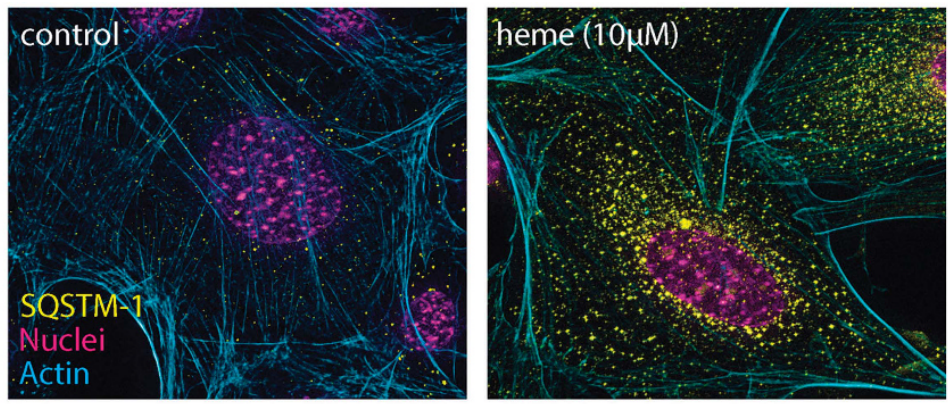

b
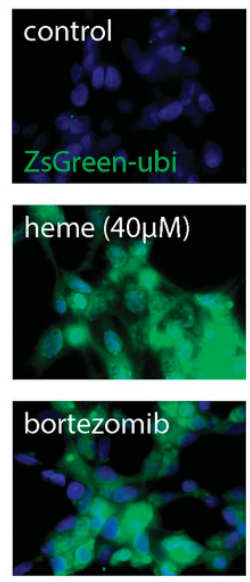

d

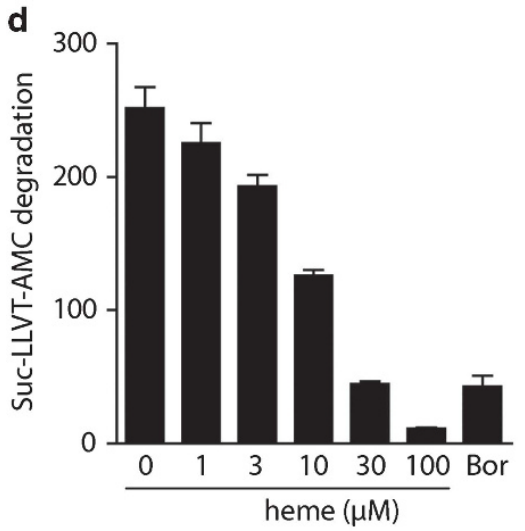

C

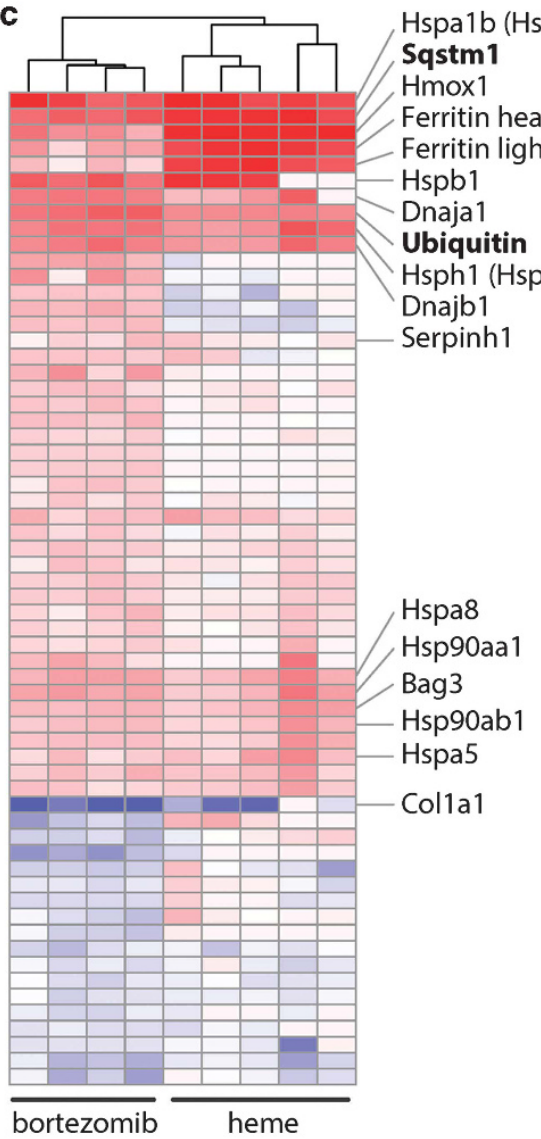

Figure 4 Heme is an inhibitor of the proteasome. (a) Immunofluorescence of Sqstm1/p62 (yellow) and actin (cyan) in Hmox1 (+/+) and Hmox1 ( - / - ) MEFs after exposure to heme $(5 \mu \mathrm{M})$ for $5 \mathrm{~h}$. Magenta indicates nuclei. Images were acquired with a Zeiss Observer Z1 equipped with an ApoTome.2 module and an Axiocam MRm at $\times 400$ original magnification. (b) Effect of heme $(40 \mu \mathrm{M})$ and bortezomib $(100 \mathrm{pM})$ treatment for $6 \mathrm{~h}$ on the accumulation of an ubiquitin-tagged fluorescent protein (ZsGreen) expressed in HEK-293 cells. (c) Hierarchical clustering analysis of all proteins that have been identified as differentially expressed in heme-treated Hmox1 $(-/-)$ cells and bortezomib-treated $(10 \mathrm{pM}) \mathrm{Hmox} 1(+/+) \mathrm{MEF}$ cells, respectively. The heme response data include all data from heme-treated Hmox $1(-/-)$ MEF cells shown in Figure 2 that were included into this analysis as the five condition group means. The bortezomib data represent data from four independent SILAC experiments. The plot includes all proteins with an absolute expression ratio of $>0.5$ (log2) in treated compared with matched untreated control cells $(P<0.05)$. Red indicates protein expression higher in treated cells, and blue indicates protein expression lower in treated cells. The identical plot with labeling of all proteins can be found in Supplementary Figure 2. (d) Enzymatic activity of purified 26S proteasome \pm increasing concentrations of heme, as assessed by monitoring the release of fluorescent AMP from the proteasome substrate peptide Suc-LLVT-Amc. Bortezomib $(B o r, 100 \mathrm{pM})$ was used as a positive proteasome inhibitor control. Data were corrected for fluorescence quenching by heme, and represent mean \pm S.D. of six independent replicates 
perinuclear region of MEF Hmox1 $(-/-)$ cells in a granular pattern. This 'aggresome' pattern of Sqstm1 accumulation was reported as a typical cellular reaction when the proteasome is inhibited. ${ }^{24}$ In addition, a short-lived fluorescent ubiquitin-conjugated ZsGreen protein (ZsGreen Proteasome Sensor Cell Line) accumulated in HEK-293 cells that were treated with either heme or with the specific proteasome inhibitor bortezomib (Figure 4b). The proteasome inhibitor function of heme is supported further by the observation that the heme-induced protein expression signature in Hmox1 $(-/-)$ MEF cells overlapped broadly with the proteome pattern of bortezomib-treated cells (Figure 4c). The overall correlation of the expression changes of all proteins that were significantly changed by either heme or bortezomib (log2 fold change $>0.5 ; P<0.05)$ was $r$ (Pearson) $=0.69(P<0.001)$. Interestingly, besides the common cluster of upregulated proteins, which includes Sqstm1, ubiquitin, and a number of heat shock and oxidative defence proteins, we also observed that heme strongly suppressed the abundance of collagen (Col1a1) 1 protein. Suppression of type 1 collagen synthesis appears to be an important effect of proteasome inhibitors that might be of therapeutic significance in fibrosing diseases. $^{25}$ Finally, using a biochemical proteasome chymotrypsin-like activity assay, we confirmed that heme dose dependently impaired cleavage of the 7-amino-4methylcoumarin (AMC)-labeled proteasome substrate peptide Suc-LLVT-AMC by the $26 \mathrm{~S}$ proteasome (Figure $4 \mathrm{~d}$ ).

Collectively, these data support that heme is an inhibitor of proteasome activity in different cell types and in a cell-free system. To explore whether the observed inhibition of the proteasome function by heme includes a direct molecular interaction between heme and the proteasome protein complex, we performed specific binding studies with UV/Vis spectrophotometry, circular dichroism (CD), and surface plasmon resonance (SPR).

Heme binds to the proteasome. The addition of proteasome-enriched cytoplasmic protein fraction to an aqueous solution of heme induced two specific spectral changes within the Soret band region of the heme UV/Vis spectrum (Figure 5): (1) a decrease of the initial broad intensity of the Soret band at $405 \mathrm{~nm}$ and (2) a bathochromic shift of the Soret band with an increased intensity at $413 \mathrm{~nm}$ (Figure 5b). In contrast to the broad band over 370-390 nm that gradually decreases in aqueous heme solutions (Figure 5a), this newly formed $\lambda_{\max }$ at $413 \mathrm{~nm}$ remained stable over days. This suggested that heme had transferred to one or multiple binding sites and stabilized within a coordination of amino acid residues (e.g., histidine and tyrosine). The $20-23 \mathrm{~nm}$ bathochromic shift of heme complexes within the protein was significantly greater than the $9-10 \mathrm{~nm}$ red shift known for heme complexes with albumin. Heme binding with formation of the $\lambda_{\max } 413 \mathrm{~nm}$ proceeded slowly, suggesting conformational changes accompanying heme accommodation in the protein. The heme titration shown in Figure $5 \mathrm{c}$ was conducted until no further increase in the absorption intensity at $413 \mathrm{~nm}$ over the intensity at $370 \mathrm{~nm}$ was observed. Heme concentrations within the range of our cell culture studies resulted in a linear increase of the Soret band intensity.
The significant bathochromic shift of the Soret band and the time-dependent adjustment of its intensity suggested heme coordination with specific amino acid residues in the protein. We therefore evaluated the protein-induced conformational changes of the heme molecules using CD (Figure 6a). In isolation, neither the proteasome protein fraction nor heme exhibited optical activity in the visible range (green and gray traces at the zero line). However, heme complexes with proteasome protein fractions F370 (and F360; not shown) demonstrated clear $C D$ spectra. Heme at $4 \mu \mathrm{M}$ in the proteasome fraction (blue trace) clearly exhibited optical activity at $414 \mathrm{~nm}$ (optically active Soret band) and at $392 \mathrm{~nm}$ (presumably a heme dimer), both related to the heme absorption spectrum (red overlay of absorbance). Appearance of these two Cotton Effects (CE) in the visible (heme-related) range of the $C D$ spectrum indicated protein-induced chirality that is typical for complex formation. Whereas the intensities of the CEs at $4 \mu \mathrm{M}$ heme content were relatively low, the CD spectra at higher ligand concentrations showed much stronger intensities and more induced CD bands. The CD spectrum of the protein sample containing $30 \mu \mathrm{M}$ heme (black trace) shows several relatively strong CEs in the $350-450 \mathrm{~nm}$ range, strongly suggesting that the higher concentration of heme allowed the ligand to occupy (multiple) binding sites of lower affinity (or accessibility) in addition to the primary binding sites.

Whereas the complementary UV/Vis spectrum showed a broad absorption band because of overlapping spectra of several species including nonbound heme or hemeassociated with the protein surface without being distorted, the CD spectra provided additional insight specific to heme molecules internally bound and distorted by the coordination with protein chiral binding sites. The Soret region CD bands observed for $30 \mu \mathrm{M}$ heme/protein complexes included strong negative CE at $402 \mathrm{~nm}$, two positive CEs at 410 and $419 \mathrm{~nm}$, and small negative at $432 \mathrm{~nm}$. Such a pattern suggested heme binding to more than one binding site, with the split (positive/ negative) profile indicating a possible exciton coupling between two or more heme molecules located in close vicinity. The bands observed for this sample in the $350-390 \mathrm{~nm}$ region including CEs at 386, 375, and $359 \mathrm{~nm}$, suggested other heme species (e.g., heme dimer) may be bound, causing conformational distortion within certain protein cavities.

As the proteasome protein preparations used for the above studies contained small amounts of other cytoplasmic proteins in addition to the $26 \mathrm{~S}$ proteasome, we confirmed the specificity of heme binding to the proteasome by SPR analysis using a highly purified $26 \mathrm{~S}$ proteasome preparation (Figure 6b). Sensograms of a heme concentration series $(0-60 \mu \mathrm{M})$ injected over a channel with immobilized $26 \mathrm{~S}$ proteasome (right) showed a concentration-dependent binding response. No binding could be observed with the same heme concentration series on a negative control channel of the same chip surface that had been EDC/NHS activated and ethanolamine deactivated but not been protein covered (left).

Heme stress causes accumulation of lipid peroxidemodified proteins in HMOX1 $(-I-)$ cells. The second heme exposure response that was identified in our protein expression data analysis was related to oxidative stress 

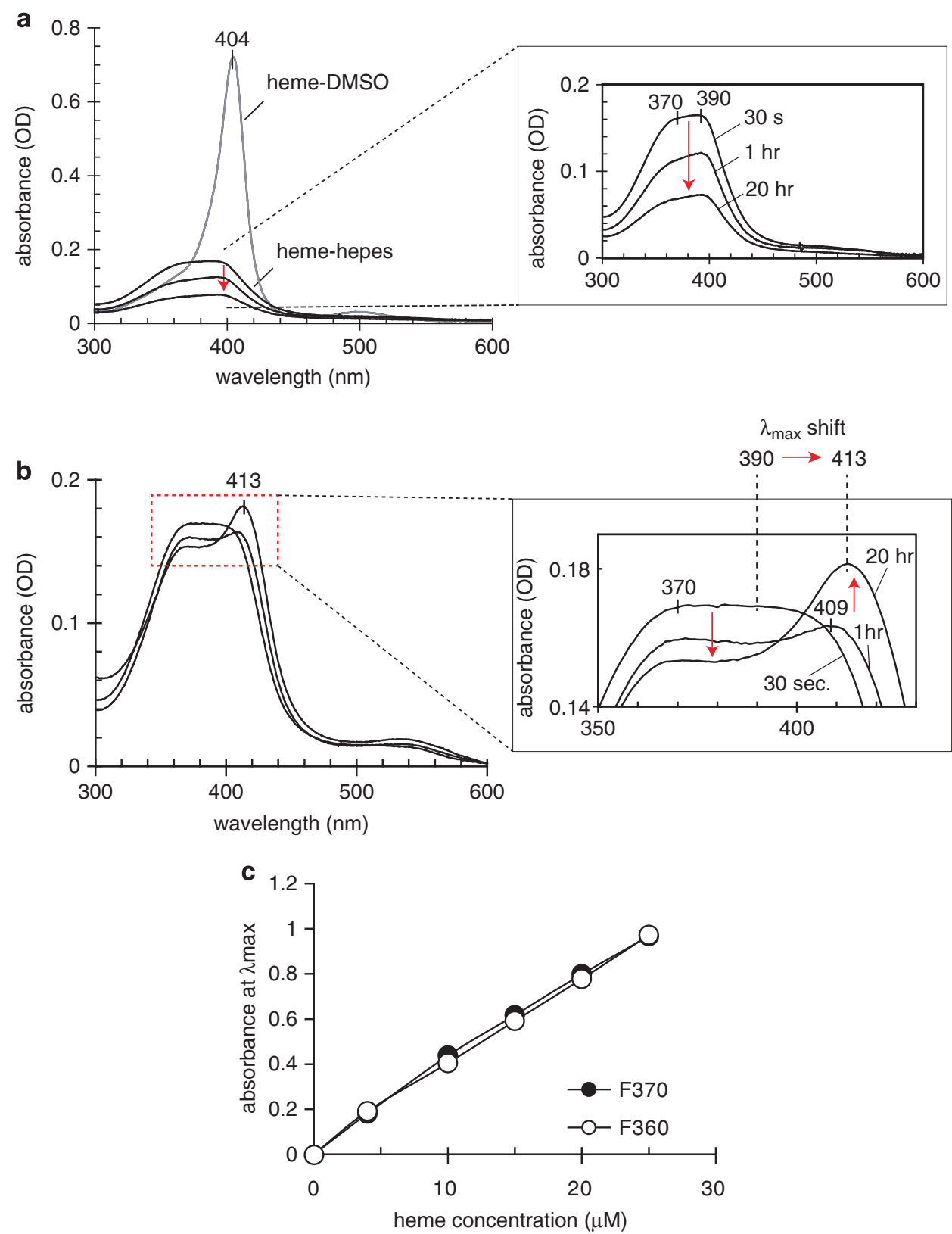

Figure 5 Heme binding to proteasome: UV/Vis spectrophotometry. (a) UV/Vis spectra of heme in DMSO or diluted in Hepes buffer (pH 7.4). The inset shows the timedependent absorbance decay (red arrow) of heme in HEPES buffer. The spectra of heme in DMSO remained stable over days. (b) Time-dependent changes in the UV/Vis spectra of the heme in HEPES buffer sample shown in (a) after addition of proteasome-enriched cytoplasmic protein fraction. The inset shows enlarged selected spectra that reflect the major changes over time. The protein concentration in the proteasome fraction was $1.4 \mathrm{mg} / \mathrm{ml}$. Identical results were observed with two proteasome fractions from rabbit reticulocyte (F360) and from HeLa cells (F370), respectively. (c) Changes in maximum absorbance after heme binding to the two proteasome fractions F360 and F370 across a range of heme concentrations

defense. Accordingly, higher levels of lipid peroxide-protein adducts were detected in heme-treated Hmox1 $(-/-)$ than in Hmox $1(+/+)$ MEF cells when the cells were heme exposed in the presence of an alkyne-tagged analog of linoleic acid, which is an unsaturated, heme-reactive fatty acid (Supplementary Figure 3). The accumulation of lipid peroxide-adducted proteins in this model is likely indicative of a combined result of heme-triggered peroxidative reactions and impaired reparative proteasome function in hemestressed cells, respectively.
Differentiation of proteasome inhibition and oxidative reactivity as independent effects of free heme. To experimentally differentiate the contributions of oxidative processes that could lead to protein modifications and oxidation-independent proteasome inhibitor activity of heme, we performed a screening of different metal porphyrins with the aim of identifying non-iron porphyrins with significant proteasome inhibitor activity, but absent oxidative activity. We found that among the tested metal porphyrins, cobalt protoporphyrin (CoPP), similar to heme, increased Sqstm1 

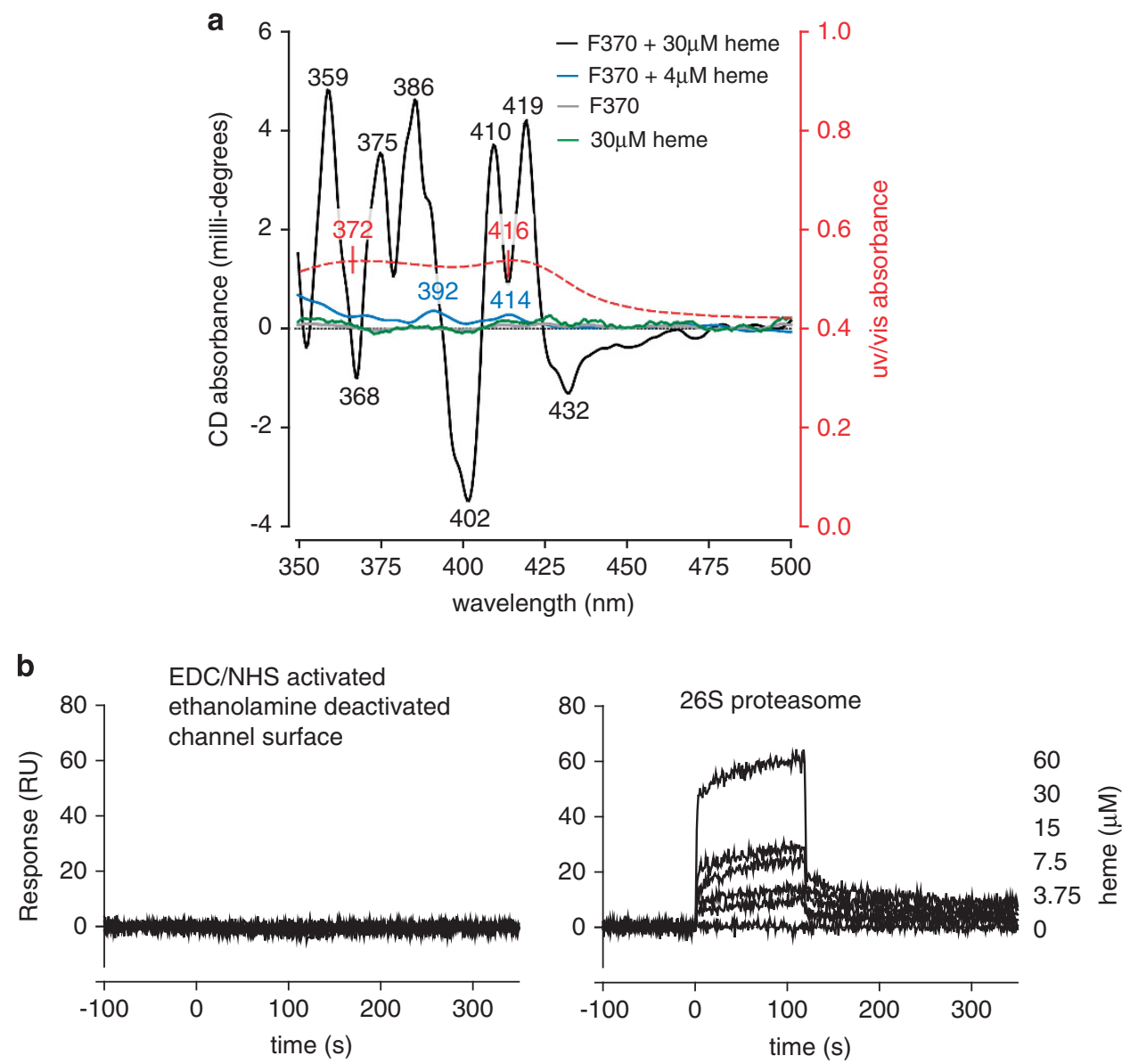

Figure 6 Heme binding to proteasome: circular dichroism and surface plasmon resonance. (a) CD of proteasome fraction F370, $6.6 \mathrm{mg} / \mathrm{ml}$ protein content (gray trace), heme, $30 \mu \mathrm{M}$ (green trace), and $4 \mu \mathrm{M}$ heme in the F370 proteasome fraction is shown (blue trace). The optical activities observed at 414 and at $392 \mathrm{~nm}$ (blue trace) are both related to the heme absorption spectrum (red overlay of absorbance). These two CEs in the visible range of the CD spectrum indicated protein-induced chirality. The CD spectrum of the F370 fractione containing $30 \mu \mathrm{M}$ heme (black trace) shows several relatively strong CES in the 350-450 nm range, indicative of multiple heme binding sites. (b) Surface plasmon resonance measurements of heme binding to $26 \mathrm{~S}$ proteasome. Sensograms of a heme concentration series $(0-60 \mu \mathrm{M})$. (Left panel) Negative control channel that had been EDC/ NHS activated and ethanolamine deactivated but not been protein covered (left). (Right panel) Channel with immobilized 26S proteasome showing a concentration-dependent binding response

protein and caused accumulation of ubiquitinated proteins in Hmox1 $(-/-)$ MEFs (Figure 7a). Interestingly, in CoPPtreated cells, the accumulated Sqstm1 appeared only in the expected monomeric (62 kDa) form, whereas higher molecular forms that likely represent oxidized and crosslinked protein were found in heme-treated cells. This observation already indicated that CoPP might be a proteasome inhibitor that could lead to the accumulation of ubiquitin-tagged proteins but does not share the oxidative (protein modifying) reactivity of heme. Like heme, CoPP also caused accumulation of Sqstm1-myc in Sqstm1-myc-transfected RAW264 cells (Figure $7 \mathrm{~b}$ ) and it significantly inhibited cleavage of Suc-LLVT-AMC by purified 26S proteasome (Figure 7c). In contrast to FePP, however, CoPP did not oxidize linoleic acid (Figure 7d) and less lipid-product adducts were detected in CoPP- compared with FePP-treated Hmox1 (-/-) MEFs (Figure 7e). Furthermore, FePP, not CoPP, was found to deplete reduced glutathione $(\mathrm{GSH})$ in $\mathrm{Hmox} 1(-/-)$ MEFs (Figure 7f).

We further demonstrate that FePP/heme also inhibits the proteasome independent of oxidant stress. The proteasome inhibitory activity of FePP/heme was not impaired by the antioxidant Trolox (6-hydroxy-2,5,7,8-tetramethylchroman-2-carboxylic acid). In contrast, at an equal antioxidant concentration, Trolox completely blocked heme-triggered lipid peroxidation (measured as production of thiobarbituric acid reactive substances (TBARS)) in soybean lecithin micelles (Figure $7 \mathrm{~g}$ ). Identical results were obtained with the antioxidant dithiothreitol (DTT) (data not shown).

Collectively, the above experiments suggest that heme could inhibit the proteasome by a mechanism independent of its specific oxidative effect.

During single-agent exposure, heme was substantially more toxic to Hmox1-deficient cells than CoPP or the selective nonporphyrin proteasome inhibitor bortezomib (Supplementary Figure 4). This suggests that although proteasome inhibition by heme is at least in part independent of its oxidative reactivity, the oxidative reactivity of the iron protoporhyrin is an essential component of cytotoxicity. Likely, proteasome impairment could exacerbate toxicity by promoting accumulation of oxidized and toxic proteins. 


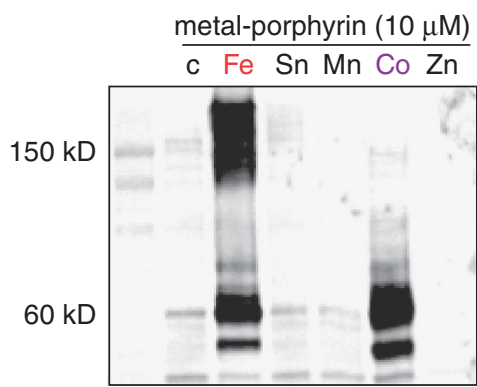

Sqstm1

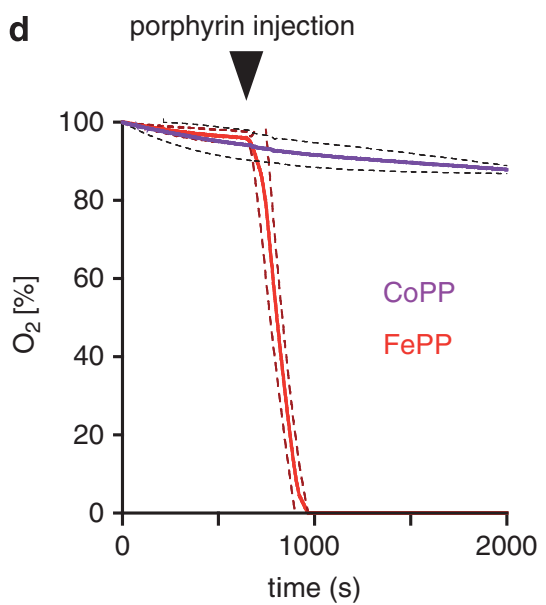

\section{g}

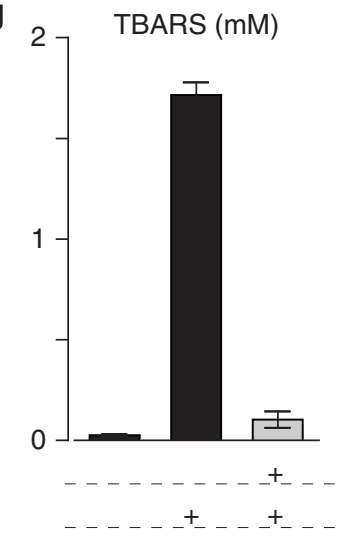

metal-porphyrin $(10 \mu \mathrm{M})$

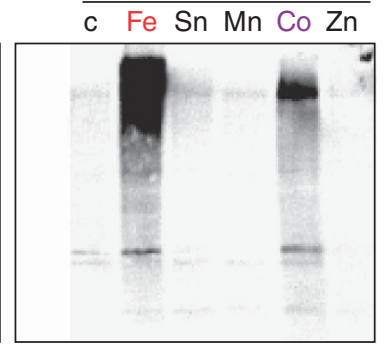

ubiquitin

b

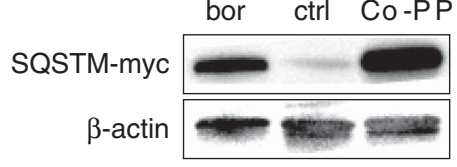

C

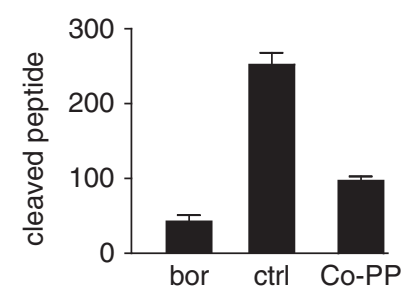

e porphyrin

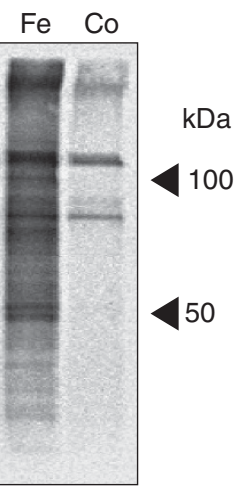

f
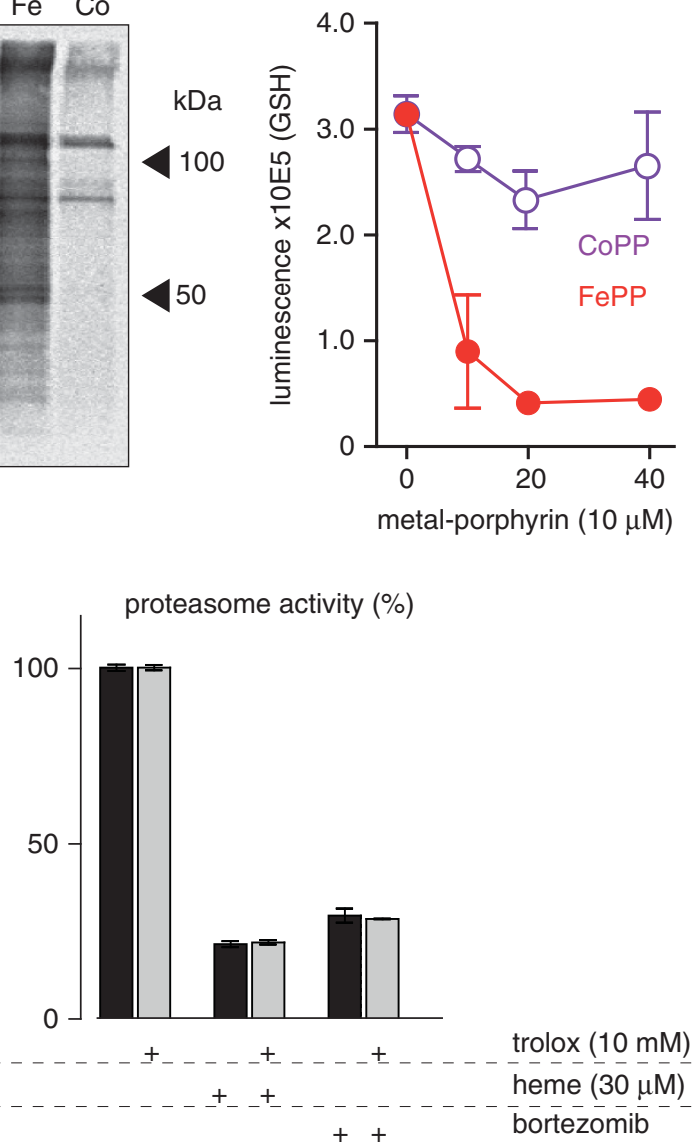

Figure 7 Dissection of proteasome inhibitor and oxidative activities of heme. (a) Hmox1 ( $/-$ ) MEFs were incubated with different metal porphyrins at $10 \mu \mathrm{M}$ for $18 \mathrm{~h}$. Expression of Sqstm1 and ubiquitin protein was probed by western blot. Accumulation of Sqstm1 and high-molecular-weight ubiquitin protein aggregates in cells treated with FePP and CoPP (Fe: iron; Sn: tin; Mn: manganese; Co: cobalt; Zn: zinc). (b) Accumulation of constitutively expressed SQSTM-myc in RAW264 cells after treatment with CoPP and bortezomib (Bor, $100 \mathrm{pM}$ ) for $12 \mathrm{~h}$ (western blot against myc-tag; $\beta$-actin as a loading control). (c) Inhibition of the proteolytic activity of purified $26 \mathrm{~S}$ proteasome by CoPP and bortezomib was quantified by monitoring the release of fluorescent AMP from the proteasome substrate peptide Suc-LLVT-Amc. All data for CoPP were corrected for fluorescence quenching by the porphyrin. Data represent mean \pm S.D. of six biologic replicates. (d) Linoleic acid oxidation by FePP but not by CoPP. Equal amounts of each porphyrin (final concentration $10 \mu \mathrm{M}$ ) were injected into stirred, airtight reaction vessels at $37^{\circ} \mathrm{C}$ containing linoleic acid as the reactant. Oxygen concentration was measured continuously during the reaction with a Clark type electrode. Data represent mean \pm S.D. (dashed lines) of three independent experiments. (e) Formation of cellular protein-lipid adducts in FePP. treated $(20 \mu \mathrm{M}, 5 \mathrm{~h})$ or CoPP-treated $(20 \mu \mathrm{M}, 5 \mathrm{~h}) \mathrm{Hmox} 1(-/-)$ MEFs detected by click chemistry facilitated biotinylation of cell lysates and subsequent western blot detection by HRP streptavidin. (f) Glutathione (GSH) in Hmox1 ( - / - ) MEF cells after incubation with different concentrations of FePP and CoPP for $12 \mathrm{~h}$. Data indicate mean \pm S.D. of luminescence from six biologic replicates. (g, left) Heme $(30 \mu \mathrm{M})$-triggered lipid peroxidation (TBARS) in soybean lecithin micelles and inhibition by Trolox (10 $\mathrm{mM})$. Data represent mean \pm S.D. of three biologic replicates. (g, right) Enzymatic activity of purified $26 S$ proteasome as assessed by monitoring the release of fluorescent AMP from the proteasome substrate peptide Suc-LLVT-Amc in the presence or absence of heme $(30 \mu \mathrm{M})$, bortezomib $(100 \mathrm{pM}) \pm$ the antioxidant Trolox (10 mM). Data were corrected for fluorescence quenching by heme, and represent mean \pm S.D. of six independent replicates 
a
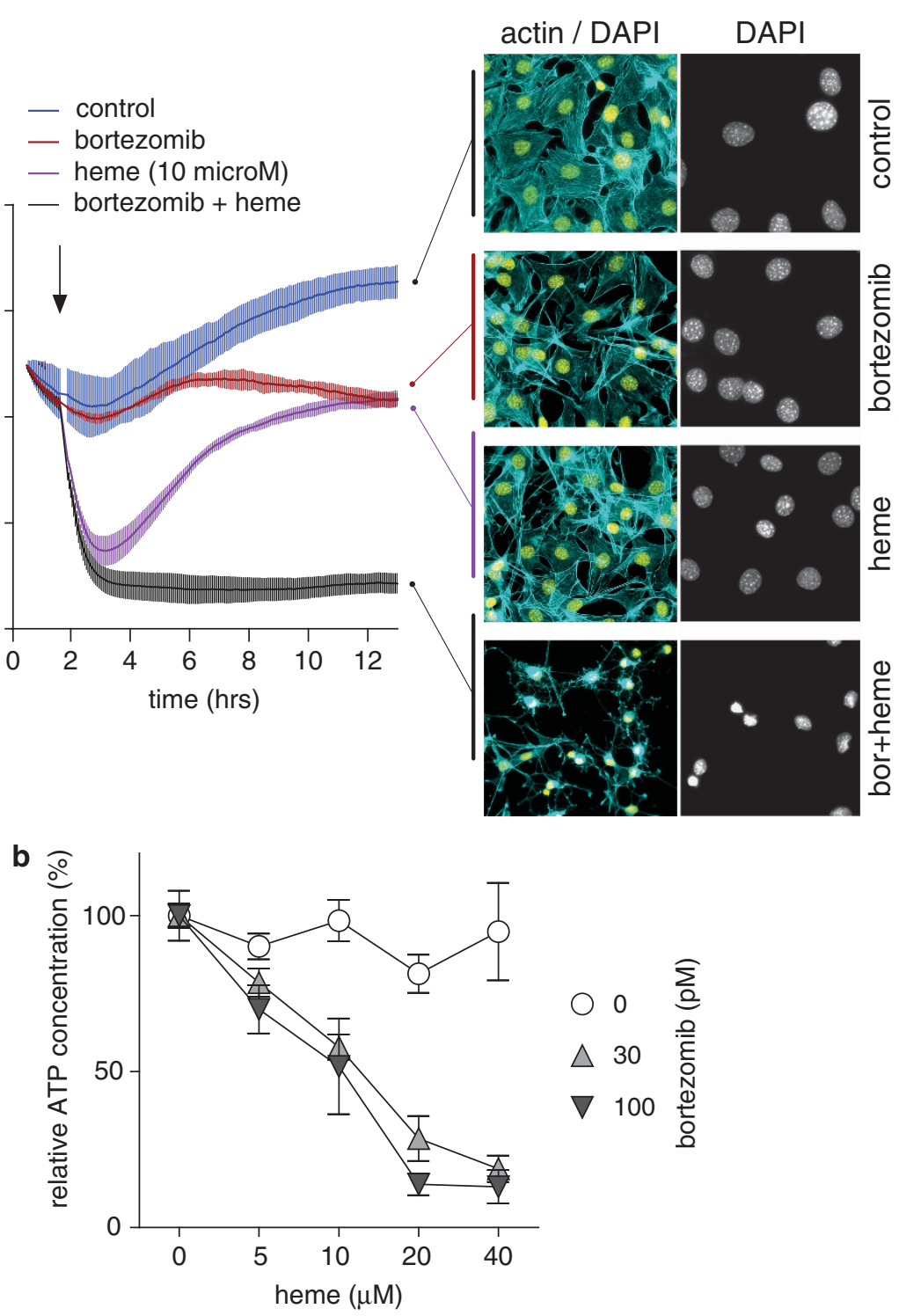

Figure 8 Proteasome inhibition sensitizes cells for heme toxicity. (a) Monolayer resistance of confluent Hmox1 (+/+) MEF cells was measured with an ECIS instrument during incubation of the cells with or without heme $(10 \mu \mathrm{M})$, bortezomib $(100 \mathrm{pM})$, or the combination of heme+bortezomib. Data represent mean \pm S.D. of four biologic replicates. At the end of the experiment, cells were fixed and stained for fluorescence microscopy with DAPI (yellow: nuclei) and Alexa 488 phalloidin (cyan: actin cytoskeleton). (b) Hmox $1+(++)$ MEF cells were treated with a range of heme concentrations in the presence or absence of bortezomib ( $30 \mathrm{pM}$ and $100 \mathrm{pM}$ ). After $12 \mathrm{~h}$, cellular ATP was measured with a luminescence assay. Data are normalized to the respective control and represent mean \pm S.D. of six biologic replicates

To support this hypothesis, we studied whether proteasome inhibition by bortezomib could make the more heme-resistant Hmox1 (+/+) MEF cells more sensitive to the impact of heme toxicity. Figure $8 \mathrm{a}$ shows monolayer integrity measured with an Electric Cell-substrate Impedance Sensing (ECIS) system (ibidi GmbH, Planegg/ Martinsried, Germany) instrument over time as well as end point fluorescence images of Hmox1 (+/+) MEF cells after stimulation with bortezomib, heme $(10 \mu \mathrm{M})$, or bortezomib+heme. As noticed in the experiment shown in Supplementary Figure 4, bortezomib treatment alone shows only very moderate changes in monolayer integrity and cell morphology. Single heme exposure triggers a rapid decline in monolayer integrity (maximum effect after 2-4 h) with recovery to almost baseline level after $12 \mathrm{~h}$. No nuclear condensation could be observed at the end of the experiment. This transient effect may be caused by an early oxidative insult that is subsequently repaired after induction of high Hmox1 activity. In contrast, the concurrent treatment with bortezomib and heme caused a rapid and irreversible destruction of the cell monolayer. After $12 \mathrm{~h}$, cellular morphology was characterized by complete destruction of the cytoskeleton and nuclear condensation. This pattern of combined bortezomib+heme toxicity in Hmox1 (+/+) MEF cells was also observed in independent experiments where decreased cellular ATP was measured as a marker of toxicity over a range of heme concentrations in the presence or absence of bortezomib (Figure 8b). 


\section{Discussion}

The results of our studies support a model whereby high levels of intracellular heme disrupt cellular homeostasis by the combined activities of proteasome inhibition and oxidative damage. Proteasome inhibition promotes accumulation of damaged proteins and may thereby critically enhance the cellular injury that is triggered by oxidative heme effects.

Tightly balanced control of heme exposure, metabolism, and cellular repair is essential to prevent heme toxicity. Under physiologic conditions a large metabolic flux continuously detoxifies $\mathrm{Hb}$-derived heme by heme oxygenases, a function that is particularly effective in professional heme-catabolizing cells such as macrophages. However, during severe hemolysis, bleeding, or rhabdomyolysis, the limited heme scavenging and detoxifying capacity of tissues may be overwhelmed by large quantities of free heme, and heme-triggered toxicity may result. Acute renal injury in cases of severe hemoglobinuria or myoglobinuria and neuronal toxicity in cases of intracerebral or subarachnoid hemorrhage are examples of such disease states.

In our studies, we used a systematic approach to obtain a comprehensive overview of the adaptive and disruptive cellular processes that are triggered by heme levels exceeding the homeostatic range. We used a cell culture model of extracellular heme exposure combined with manipulated Hmox1 expression to monitor cellular responses across a range of heme exposures from physiologic to subtoxic to overt toxicity. A network analysis generated from the protein expression profiling data revealed a compelling picture of the metabolic toxicity of heme, leading to this novel model.

The pro-oxidant activity of heme, which was evident in our experiments, is well known and has been reported previously as a cause of heme-mediated cytotoxicity. ${ }^{11,26}$ In our studies, direct evidence for the oxidative toxicity of heme was provided by the observations that heme exposure depleted cellular GSH, caused accumulation of oxidized lipid peroxide-protein adducts and, eventually, triggered expression of oxidative defense proteins. In addition to this well-established role of heme as an oxidant, a novel and consistent finding of the present study is that excessive heme levels lead to dysfunctional cellular protein degradation by the proteasome.

The most direct evidence for an adverse impact of heme on cellular protein homeostasis was provided by the observation that several heat shock proteins, ubiquitin, and the ubiquitin adaptor protein Sqstm1 were found among the primary proteins that accumulated in heme-stressed cells. Subsequent mechanistic studies traced these effects to impaired proteasome function. Heme-impaired proteasome function was evident in our screening cell type as well as in a murine macrophage cell line and in human HEK-293 cells, and this effect was confirmed in cell-free protein binding and peptide degradation studies.

The proteasome is the principal pathway to remove senescent and damaged proteins, and intact proteasome function is essential to preserve and repair cellular homeostasis during oxidative stress, such as that triggered by heme exposure. ${ }^{27}$ Impaired proteasome function during heme stress may therefore exacerbate heme toxicity, as oxidized and potentially toxic proteins could accumulate. In line with this hypothesis, we showed that proteasome inhibition by nonoxidative inhibitors, such as bortezomib or CoPP, was not sufficient to trigger apoptotic cell death per se. However, proteasome inhibition by bortezomib drastically enhanced the susceptibility of cells to concurrent heme stress.

Several mechanisms could contribute to the impaired regulation of protein homeostasis in heme-overloaded cells. First, we provide direct biochemical evidence that heme binds to and inhibits the proteasome. This inhibitor activity appears to be, at least in part, independent of the pro-oxidant activity of heme because the redox-inert CoPP also functions as a proteasome inhibitor and the antioxidant trolox could not antagonize heme's proteasome inhibitor activity at high concentrations that completely blocked lipid peroxidation. As a second mechanism, heme-triggered oxidative reactions or lipid peroxidation products could structurally modify and damage the proteasome itself. ${ }^{28}$ Finally, oxidized, crosslinked, and aggregated proteins such as the high molecular forms of Sqstm1 found in heme-stressed cells in our studies are poor proteasome substrates and may interfere negatively with proteasome function. ${ }^{28}$ It is likely that all three mechanisms in addition to increased transcription following heme stress contributed to the dramatic accumulation of proteasome substrates that accompanied heme toxicity in our studies.

Proteasome inhibition has not been explored as an adverse metabolic function of heme thus far, although this concept is consistent with older biochemical observations. Before the widespread use of highly specific peptide-based proteasome inhibitors, such as MG-132 or bortezomib, heme was used empirically to block proteolysis by the proteasome. ${ }^{19-23}$ In these older studies, evidence for the proteasome inhibition by heme can be found across a broad range of biochemical systems. The inhibitor concentrations reported in those studies were within the range used in our experiments. The principal target of heme within the proteasome was identified as the 19S ATPase subunit of the $26 \mathrm{~S}$ complex. ${ }^{29}$ In addition, more recent pharmaceutical developments identified chemically modified porphyrins as a new class of potent proteasome inhibitors. $^{30}$

In conclusion, our studies identified heme as an endogenous proteasome inhibitor molecule. In states of intracellular heme, excess proteasome inhibition acts in concert with the pro-oxidant activity of the iron protoporphyrin, resulting in the accumulation of damaged proteins that contribute to the triggering of cell death.

\footnotetext{
Materials and Methods

Generation and isotopic labeling (SILAC) of MEFs. Hmox $1(+/+)$ and Hmox1 ( - / - ) MEFs were obtained from breeding Hmox1 $(+/+) \times H \operatorname{mox} 1(+/+)$ and Hmox1 $(-/-) \times H_{m o x} 1(-/-)$ mice (BALB/c background), respectively, with approval from the Animal Ethics Committee of the University of Sydney. The 3T3 protocol was used initially, and spontaneously immortalized cells with stable growth rate were then established. The growth rate of Hmox 1 ( $-/-)$ MEFs was $\sim 50 \%$ of that of Hmox1 $(+/+)$ MEFs. Hmox1 $(-/-)$ MEFs were confirmed to be deficient in the Hmox1 protein by western blotting, and Hmox1 $(+/+)$ and Hmox1 $(-/-)$ MEFs contained comparable amounts of tubulin-standardized Hmox2 protein (data not shown).

Wild-type $(H \operatorname{mox} 1+/+)$ and Hmox1 knockout (Hmox1 - / - ) MEFs were grown in a DMEM medium (GIBCO, Basel, Switzerland) containing 10\% FBS, penicillin/ streptomycin (GIBCO), and $2 \mathrm{mM}$ L-glutamine supplemented with $0.084 \mathrm{mg} / \mathrm{ml}$ ${ }^{13} \mathrm{C} 6-{ }^{15} \mathrm{~N}$ (medium), or ${ }^{13} \mathrm{C} 6-{ }^{15} \mathrm{~N} 4 \mathrm{~L}$ (heavy) L-arginine and $0.146 \mathrm{mg} / \mathrm{ml}{ }^{13} \mathrm{C} 6-{ }^{15} \mathrm{~N}$
} 
(medium) or ${ }^{13} \mathrm{C} 6-{ }^{15} \mathrm{~N} 2$ (heavy) L-Lysine, or with the corresponding nonlabeled amino acids (light), respectively. Using mass spectrometry, the labeling efficiency was confirmed to be $>99 \%$. Individual SILAC experiments were always performed with cells of the same genotype and included a control and two treatment conditions (different heme concentrations) in $\mathrm{L}-, \mathrm{M}$-, and $\mathrm{H}$-labeled cells. The association of treatment conditions and the labeling group were permutated across replicates to exclude any labeling effects.

Assays for cell viability, apoptosis, cellular glutathione, and linoleic acid oxidation. Chemiluminescent assays were used to measure ATP (CellTiter-Glo Assay, Promega AG, Dubendorf, Switzerland), caspase 3/7 activity (Caspase-Glo 3/7 assay, Promega,) and reduced glutathione (GSH-Glo Glutathione assay, Promega). Experiments were performed in 96-well plates, and luminescence was measured with an Infinite M200-Pro plate reader (Tecan Group Ltd, Männedorf, Switzerland). Cell monolayer integrity was analyzed with the ECIS system (ibidi $\mathrm{GmbH}$ ). Oxidation of linoleic acid by porphyrin was measured in a closed system with a Clark-type $\mathrm{O}_{2}$ electrode as described earlier. ${ }^{31}$

For the quantification of nuclear condensation, MEF cells were grown on collagencoated glass coverslips. After treatment, cells were fixed with formalin and stained with Hoechst 33342 (Sigma, St. Louis, MO, USA). Random wide-field fluorescent images were acquired with a Zeiss Axioscope.2 (Carl Zeiss AG, Feldbach, Switzerland) with $\times 200$ original optical magnification. Images were analyzed with Cell Profiler image analysis software (Broad Institute; www.cellprofiler.org). A nuclear condensation score $(0-100 \%$ of nuclei) was calculated based on a multivariate model that included nuclear radius and fluorescence intensity.

\section{Mass spectrometry analysis of SILAC-labeled protein extracts}

Sample preparation: Total cellular protein was extracted using the Cellytic-M reagent (Sigma-Aldrich Chemie GmbH, Buchs, Switzerland) supplemented with the Complete Mini Protease Inhibitor (Roche Diagnostics, Rotkreuz, Switzerland). After three freeze-thaw cycles, nuclei and cellular debris were removed by a 30-min centrifugation step $(16000 \times g)$. The protein concentration of each sample was determined using BCA assay (Pierce, Fisher Scientific, Wohlen, Switzerland). Samples were normalized to $30 \mu \mathrm{g}$ and the light-, medium-, and heavy-labeled experimental samples (each with a different treatment/control condition, i.e., different heme exposure) were pooled in equal ratios. Pooled samples were precipitated by adding TCA to a final concentration of $10 \% \mathrm{w} / \mathrm{v}$ and incubated on ice for $20 \mathrm{~min}$. After centrifugation at $16000 \times g$, the supernatant was removed, and the pellets were washed three times with ice-cold acetone, dried at $95^{\circ} \mathrm{C}$ for $5 \mathrm{~min}$ and reconstituted in $0.1 \%$ RapiGest (Waters, Milford, MA, USA). Disulfide bonds were reduced by DTT additive and finally blocked by treatment with iodoacetamide. Subsequently, proteins were digested with trypsin overnight at $37^{\circ} \mathrm{C}$. Lyophilized peptide mixtures were dissolved in $10 \mathrm{mM}$ potassium diphosphate and $25 \%$ acetonitrile (ACN) for strong cation exchange (SCX) fractionation.

Sample fractionation: Labeled peptides were fractionated using SCX liquid chromatography, with a Polysulfethyl A $2.1 \mathrm{~mm} \times 200 \mathrm{~mm}, 5 \mu \mathrm{m}, 300 \AA$ column (PolyLC, Columbia, MD, USA). Solvent A was $10 \mathrm{mM}$ potassium diphosphate $(25 \%$ $\mathrm{ACN}, \mathrm{pH}<3.0$ ), and solvent $\mathrm{B}$ was $10 \mathrm{mM}$ potassium diphosphate and $500 \mathrm{mM}$ potassium chloride $(25 \% \mathrm{ACN}, \mathrm{pH}<3.0)$. The SILAC-labeled peptides were resuspended in solvent A and applied to the SCX column. Chromatography was performed at a flow rate of $0.3 \mathrm{ml} / \mathrm{min}$, according to the following gradient: 0-10 $\mathrm{min}$, $0 \%$ solvent B; $10-60 \mathrm{~min}, 0-100 \%$ solvent $B ; 60-65 \mathrm{~min}, 100 \%$ solvent $B$; $65-90 \mathrm{~min}, 0-100 \%$ solvent A. For each HPLC run, 24 out of 27 fractions were collected and evaporated partially to remove $\mathrm{ACN}$ in a vacuum concentrator. The collected fractions were resolved in $5 \% \mathrm{ACN}, 0.1 \%$ trifluoroacetic acid, and combined to eight pools and desalted with Sep Pak $\mathrm{C}_{18}$ cartridges (Waters).

Liquid chromatography coupled tandem mass spectrometry. SILAC experiments were analyzed using reversed-phase liquid chromatography nanospray tandem mass spectrometry (nanoLC-MS/MS) on an LTQ-Velos mass spectrometer (Thermo Scientific, Bremen, Germany) coupled to an Eksigent nano LC system (Eksigent Technologies, Dublin, CA, USA). Peptides were resuspended in 5\% ACN and $0.2 \%$ formic acid and separated using an ACN/water solvent system containing $0.2 \%$ formic acid with a flow rate of $250 \mathrm{n} / / \mathrm{min}$. Separation of the peptides was performed on a $10 \mathrm{~cm}$ long-fused silica column $(75 \mu \mathrm{m}$ i.d.; BGB Analytik, Boeckten, Switzerland), in-house packed with $3 \mu \mathrm{m}, 200 \AA$ pore size $\mathrm{C} 18$ resin (Michrom BioResources, Auburn, CA, USA). For elution, we used a step-gradient from 3 to $30 \%$ ACN in 62 min, followed by ramps to $50 \%$ ACN in 3 min and to $100 \%$ ACN in $2 \mathrm{~min}$, with a plateau of $100 \% \mathrm{ACN}$ for $4 \mathrm{~min}$. SILAC-labeled peptides were analyzed using mass-dependent MS/MS. One scan cycle comprised a survey full scan MS spectra from $\mathrm{m} / \mathrm{z} 300$ to 1700 , acquired in the FT-Orbitrap Velos with a resolution of $R=60000$ at $\mathrm{m} / \mathrm{z} 400$. This was followed by up to 20 sequential datadependent MS/MS scans. CID was completed with a target value of $1 \times 10^{4}$ in the linear trap. Collision energy was set to 35 . For all experiments, dynamic exclusion was used with one repeat count, $30 \mathrm{~s}$ repeat duration, $90 \mathrm{~s}$ exclusion duration, and a maximal of 500 entries on the exclusion list. The samples were acquired using internal lock mass calibration on $\mathrm{m} / \mathrm{z} 429.088735$ and 445.120025 .

\section{Peptide and protein identification and quantification by database} searching. Protein identification and quantification were performed using MaxQuant software (Version 1.2.2.5; http://www.maxquant.org). MaxQuant files were searched against the mouse IPI database (Version 3.68). The following MaxQuant search settings were used: maximum missed cleavages: 2; peptide mass tolerance: 6 p.p.m.; and fragment ion tolerance: $0.5 \mathrm{Da}$.

The following variable modifications were specified: oxidation on methionine, SILAC heavy Arginine: ${ }^{13} \mathrm{C} 6-{ }^{15} \mathrm{~N} 4$ (delta mass +10 ); SILAC heavy Lysine: ${ }^{13} \mathrm{C} 6-{ }^{15} \mathrm{~N} 2$ (delta mass +8); SILAC medium Arginine: ${ }^{13} \mathrm{C} 6-{ }^{15} \mathrm{~N}$ (delta mass +6 ); and SILAC medium Lysine: ${ }^{13} \mathrm{C} 6-{ }^{15} \mathrm{~N}$ (delta mass +6 ). Carboxyamidomethylation of cysteine was specified as fixed modification. MaxQuant data were filtered for reverse identifications (FDR set as $0.1 \%$ ) and contaminants. Data were evaluated and normalized using the Perseus software (Version 36, Max Planck Institute of Biochemistry, Martinsried, Germany). Only proteins identified by at least two unique peptides were retained for further quantitative analysis. The area under the curve (AUC) was used to determine a SILAC ratio for each peptide. The protein ratios (treatment/control) were averaged and $\log _{2}$ transformed. Further analysis (merging of data from different experiments) was performed using MATLAB (Version 7.7.0, The Mathworks Inc., Bern, Switzerland). For the statistical analysis to identify candidate proteins for differential expression, we used the one sample t-test function in R (version 3.0.2; http://www. r-project.org). Furthermore, R was used to calculate Pearson's correlation coefficient, to perform sample/protein clustering, and to draw the resulting high-level graphics. Subgroups of proteins were analyzed for enrichment of functional networks with Metacore analysis software (online version, Thomson Reuters).

Analysis of the SIM-MS2 target approach data was performed with Skyline 2.1.0 software (MacCoss Lab Software, Lab Genome Sciences, UW; https://skyline.gs. washington.edu).

Target SRM-MS2 quantification. SRM analysis was performed in cell lysates from three experiments that were independent of the SILAC discovery experiments using a modified nanoLC-MS/MS method as previously described..$^{32}$ Proteotypic peptides of Sqstm1, ubiquitin, and ferritin were monitored in targeted SIM windows over the mass range of $380-1000 \mathrm{~m} / \mathrm{z}$. All peptides have been monitored in their three different SILAC isotope-labeled masses (light, medium, and heavy). As no SCX fractionation was performed before the Target-SRM/MS2 analysis, a longer ACN gradient (from 3 to $30 \%$ in $140 \mathrm{~min}$ ) was applied to obtain an optimal number of data points along the peak elution profile for the quantification (DATA points $>10$ ). The AGC target was set to $5 \times 10^{4}$ in the FT-Orbitrap mass analyzer $(\mathrm{R}=30000)$. Targeted MS/MS spectra were acquired in the linear ion trap using a global scheduled inclusion list (IL). Every peptide in the IL was fragmented in $\mathrm{CID}$ mode $(\mathrm{CE}=35)$ within a RT window of $8 \mathrm{~min}$. Isolation window and activation time were set to $1.2 \mathrm{Da}$ and $10 \mathrm{~ms}$, respectively.

Proteasome chymotrypsin-like activity. Proteasomal chymotrypsin-like activity was measured with the Suc-Leu-Leu-Vyl-Tyr-AMC reporter substrate (Enzo Life Sciences AG, Lausen, Switzerland) at a concentration of $200 \mu \mathrm{M}$ in an assay buffer containing $50 \mathrm{mM}$ Tris- $\mathrm{HCl}$ with pH 7.6, $1 \mathrm{mM}$ DTT, $40 \mathrm{mM} \mathrm{KCl}, 5 \mathrm{mM} \mathrm{MgCl}$, and $1 \mathrm{mM} \mathrm{Na}{ }_{2}$ ATP. Proteasome 26S Fraction (Sigma) was used at a concentration of $50 \mathrm{nM}(0.1 \mathrm{~g} / \mathrm{l})$. Heme $(0-100 \mu \mathrm{M})$ or bortezomib $(100 \mathrm{pM})$ was added to the reaction mixture, and the fluorescence of free $\mathrm{AMC}$, released during degradation, recorded with an Infinite M200-Pro plate reader (Tecan) at $37^{\circ} \mathrm{C}$ every $2 \mathrm{~min}$. To compensate for AMC fluorescence quenching by the porphyrin, a distinct AMC fluorescence standard curve was constructed for every measured heme and bortezomib concentration. $V_{\max }$ was calculated by linear regression analysis of hexaplicate samples.

Construction of a myc-p62/SQSM1 RAW-264 cell line. RAW-264 cells were transfected with a pCMV6-Entry plasmid coding for Myc-DDKtagged p62/SQSM1 (RC203214, OriGene, Rockville, MD, USA) using the Amaxa Nucleofector Kit V (Lonza, Basel, Switzerland) according to the 
manufacturer's protocol. Transfected cells were passaged six times in a selection medium to produce stable transfectants.

Accumulation of ubiquitin-tagged proteins. HEK 293 ZsGreen Proteasome Sensor Cells (BD, Allschwil, Switzerland) were grown to confluence according to the manufacturer's protocol. Cells were exposed to $40 \mu \mathrm{M}$ heme or $100 \mathrm{pM}$ bortezomib for $8 \mathrm{~h}$ on glass coverslips, fixed for $15 \mathrm{~min}$ with formaldehyde (4\% in PBS), stained with DAPI, and visualized using a Zeiss Axioscope.2 (Carl Zeiss AG, Feldbach, Switzerland) with 630 times original optical magnification. Exposure time in the green channel was fixed to $10 \mathrm{~ms}$ for all images.

Heme binding to proteasome fraction by UV/Vis and circular dichroism. Protein fraction II from rabbit reticulocytes (F360) and protein fraction II from HeLa cells (F370) were purchased from Boston Biochem Inc. (Cambridge, MA, USA).

Electronic absorption (UV/Vis) measurements were performed on an Agilent HP8453 Spectrophotometer (Agilent Technologies Deutschland GmbH, Waldbronn, Germany) at $25^{\circ} \mathrm{C}$ in the range $250-700 \mathrm{~nm}$ using a quartz cuvette with a $1 \mathrm{~cm}$ path length.

Each of the stock solutions of protein fraction II from rabbit reticulocyte (F360) and from HeLa ( $\mathrm{F} 370)$ were diluted to the protein concentration of $1.4 \mathrm{mg} / \mathrm{ml}$ using $25 \mathrm{mM}$ HEPES buffer $(\mathrm{pH} 7.4)$ and were stored at $-80^{\circ} \mathrm{C}$ until use. A freshly prepared $1.5 \mathrm{mM}$ stock solution of heme in DMSO was purged with argon and kept protected from light during use for no longer than 2 days. The concentration of the heme in the stock solution was determined using an extinction coefficient at $404 \mathrm{~nm}$ $170000 \mathrm{M}^{-1} \mathrm{~cm}^{-133}$. Titration was performed stepwise by adding a $4 \mu \mathrm{l}$ aliquot of $1.5 \mathrm{mM}$ stock solution of heme to $1.5 \mathrm{ml}$ of each protein sample (F360 or F370) and conducted at $25^{\circ} \mathrm{C}$ and at $37^{\circ} \mathrm{C}$ with UV/Vis monitoring started immediately in a differential mode using the initial spectrum of the protein solution as a blank.

CD measurements were performed on a Jasco J-815 Spectropolarimeter (JASCO Co., Tokyo, Japan) at $25 \pm 0.2^{\circ} \mathrm{C}$ maintained by a Peltier thermostat. The induced CD spectra were recorded between 700 and $350 \mathrm{~nm}$ in a rectangular quartz cuvette of $10 \mathrm{~mm}$ path length and accumulated over 10 consecutive scans using a scan speed of $50 \mathrm{~nm} / \mathrm{min}$, a bandwidth of $1.0 \mathrm{~nm}$, and a resolution of $0.2 \mathrm{~nm}$. The baseline was subtracted by running HEPES buffer ( $\mathrm{pH}$ 7.4) as a blank. An ellipticity of $\mathrm{CD}$ spectra was expressed in mdeg.

SPR analyses. SPR measurements were performed on a ProteOnXPR36 instrument (Bio-Rad Laboratories, Inc., Hercules, CA, USA). GLH Sensor Chips, $N$-hydroxysuccinimide (NHS), $N$-ethyl- $\mathrm{N}^{\prime}$-(3-dimethylaminopropyl)carbodiimide (EDC), and $1 \mathrm{M}$ ethanolamine $\mathrm{HCl}, \mathrm{pH} 8.5$, were purchased from Bio-Rad. Data were collected with the biosensor instrument thermostated to $+25^{\circ} \mathrm{C}$. A solution of $0.01 \mathrm{M}$ HEPES, pH 7.6, $0.15 \mathrm{M} \mathrm{NaCl}, 0.003 \mathrm{M}$ EDTA, and $0.05 \%$ (v/v) Surfactant P20 (HBS-EP+) was used as running buffer. On a GLH chip, 630 response units $(\mathrm{RU})$ ligand and human $26 \mathrm{~S}$ proteasome (Cat. no. E-365, Boston Biochem) were immobilized by amine coupling. As a negative control and reference surface, a neighboring channel on the same chip was EDC/NHS activated and ethanolamine deactivated without protein immobilization. A concentration series of $0,3.75,7.5,15$, 30 , and $60 \mu \mathrm{M}$ heme in HBS-EP+ was injected for $120 \mathrm{~s}$ over both channels and on-spot and inter-spot data recorded.

Western blot. Samples were prepared at $5 \mu \mathrm{g}$ of total protein per well in Lämmli Buffer. The western blot protocol was described earlier and included chemiluminescence detection of the secondary HRP-labeled antibodies (Amersham, GE Healthcare, Glattbrugg, Switzerland) with the SuperSignal West Femto Maximum Sensitivity Substrate (Thermo Scientific AG). ${ }^{34}$ Images were optimized only by adjusting the brightness and contrast of the whole image using Photoshop Software (Adobe, Adobe Systems GmbH, Zurich, Switzerland). The primary antibodies were: Sqstm1: rabbit polyclonal anti-Sqstm1-Antibody (ab91526, Abcam, Cambridge, UK) at a final concentration of $0.2 \mathrm{mg} /$; Ubiquitin: rabbit polyclonal anti-Ubiquitin-Antibody (ab7780, Abcam) at a final concentration of $0.25 \mathrm{mg} /$; and Myc-Tag: rabbit polyclonal anti-Myc-Tag-Antibody (ab9106, Abcam) at a final concentration of $0.5 \mathrm{mg} / \mathrm{l}$.

Real-time PCR. Total RNA was extracted using Qiagen RNeasy kit (Qiagen, Hombrechtikon, Switzerland). Reverse transcription was performed with TaqMan reverse transcription reagents (Life Technologies, Basel, Switzerland). Real-time PCR was performed using the TaqMan Fast Advanced Master mix and TaqMan gene expression assays (Life Technologies) with FAM-labeled probes for the following targets: Sqstm1: Mm00448091_m1; Ubc: Mm02525934_g1; and Hprt:
Mm00446968_m1. Relative mRNA levels were calculated by the 7500 Fast System Sequence Detection Software Version 1.4 (Applied Biosystems, Rotkreuz, Switzerland) after normalization of each experimental sample to Hprt levels.

Detection of oxidized lipid-protein adducts. MEFs were incubated with $50 \mu \mathrm{M}$ linoleamide alkyne (LAA; Life Technologies) \pm heme for $5 \mathrm{~h}$. For immunofluorescence detection, cells were fixed with $4 \%$ paraformaldehyde in PBS, permeabilized with $0.5 \%$ Triton $X-100$, and blocked with $1 \%$ BSA before alkyne lipid-conjugated proteins were labeled with Alexa-488 azide by the Click chemistry reaction according to the protocol provided by the manufacturer (Life Technologies). For gel separation and western blot detection of conjugated proteins, MEFs were lysed with CellLytic M (Sigma). After centrifugation at $16000 \times g$ for $15 \mathrm{~min}$, protein concentrations in the cytoplasmic fractions were determined with the Pierce BCA protein assay kit (Thermo Scientific AG). Equal quantities of total cytoplasmic protein were labeled with biotin azide by the Click chemistry reaction according to the manufacturer's protocol (Jena, Bioscience GmbH, Germany). Biotinylated protein adducts were resolved and detected by SDS-PAGE and western blot using streptavidin-HRP at a dilution of $1: 4000$ (Thermo Scientific AG) followed by chemiluminescence detection with the SuperSignal West Femto Maximum Sensitivity Substrate (Thermo Scientific AG).

Measurement of TBARS. A total of $100 \mu \mathrm{l}$ of the sample was diluted in $500 \mu \mathrm{l}$ of solution of $750 \mathrm{mM}$ trichloracetic acid in $1 \mathrm{M} \mathrm{HCl}$. After vortexing, $400 \mu \mathrm{l}$ of a $25 \mathrm{mM}$ solution of 2-thiobarbituric acid in $1 \mathrm{M} \mathrm{NaOH}$ were added and the sample incubated for $60 \mathrm{~min}$ at $80^{\circ} \mathrm{C}$. After pelleting debris, TBARS were quantified in the supernatant by spectrophotometry (extinction coefficient $0.156 \mu \mathrm{M}^{-1} \mathrm{~cm}^{-1}$ ).

\section{Conflict of Interest}

The authors declare no conflict of interest.

Acknowledgements. This work was supported by the Swiss National Science Foundation (Grants 310030/120658 and 31003A/138500 to DJS); the University of Zurich Research Priority Program 'Integrative Human Physiology'; the Swiss Federal Commission for Technology and Innovation (CTI); the 'Patient Blood Management' Program of the Department of Health of the Canton of Zurich, Switzerland; and the National Health \& Medical Research Council (NHMRC) of Australia to RS. ML was also supported by a short-term visiting fellowship from the Swiss National Science Foundation.

\section{Author contributions}

FV designed the study, performed experiments, and wrote the paper; JD, LO, CAS, MP, ML, WE, SS, and EK: performed experiments and analyzed data; DRS, RS, PWB, and DJS: designed the study, analyzed data, and wrote the paper.

1. Schaer DJ, Buehler PW. Cell-free hemoglobin and its scavenger proteins: new disease models leading the way to targeted therapies. Cold Spring Harb Perspect Med 2013; 3: 1569-1579.

2. Balla G, Vercellotti G, Eaton JW, Jacob HS. Heme uptake by endothelium synergizes polymorphonuclear granulocyte-mediated damage. Trans Assoc Am Physicians 1990; 103: 174-179.

3. Schaer DJ, Buehler PW, Alayash Al, Belcher JD, Vercellotti GM. Hemolysis and free hemoglobin revisited: exploring hemoglobin and hemin scavengers as a novel class of therapeutic proteins. Blood 2013; 121: 1276-1284.

4. Boretti FS, Buehler PW, D'Agnillo F, Kluge K, Glaus T, Butt Ol et al. Sequestration of extracellular hemoglobin within a haptoglobin complex decreases its hypertensive and oxidative effects in dogs and guinea pigs. J Clin Invest 2009; 119: 2271-2280.

5. Balla J, Jacob HS, Balla G, Nath K, Vercellotti GM. Endothelial cell heme oxygenase and ferritin induction by heme proteins: a possible mechanism limiting shock damage. Trans Assoc Am Physicians 1992; 105: 1-6.

6. Nath KA, Balla G, Vercellotti GM, Balla J, Jacob HS, Levitt MD et al. Induction of heme oxygenase is a rapid, protective response in rhabdomyolysis in the rat. J Clin Invest 1992; 90: 267-270.

7. Balla J, Jacob HS, Balla G, Nath K, Eaton JW, Vercellotti GM. Endothelial-cell heme uptake from heme proteins: induction of sensitization and desensitization to oxidant damage. Proc Natl Acad Sci USA 1993; 90: 9285-9289.

8. Jeney V, Balla J, Yachie A, Varga Z, Vercellotti GM, Eaton JW et al. Pro-oxidant and cytotoxic effects of circulating heme. Blood 2002; 100: 879-887. 
9. Abraham NG, Kappas A. Pharmacological and clinical aspects of heme oxygenase. Pharmacol Rev 2008; 60: 79-127.

10. Yachie A, Niida $Y$, Wada $T$, Igarashi $N$, Kaneda $H$, Toma $T$ et al. Oxidative stress causes enhanced endothelial cell injury in human heme oxygenase-1 deficiency. J Clin Invest 1999; 103: $129-135$

11. Higdon AN, Benavides GA, Chacko BK, Ouyang X, Johnson MS, Landar A et al. Hemin causes mitochondrial dysfunction in endothelial cells through promoting lipid peroxidation: the protective role of autophagy. Am J Physiol Heart Circ Physiol 2012; 302: H1394-H1409.

12. Lin S, Yin Q, Zhong Q, Lv FL, Zhou Y, Li JQ et al. Heme activates TLR4-mediated inflammatory injury via MyD88/TRIF signaling pathway in intracerebral hemorrhage. $J$ Neuroinflammation 2012; 9: 46

13. Fortes GB, Alves LS, de Oliveira R, Dutra FF, Rodrigues D, Fernandez PL et al. Heme induces programmed necrosis on macrophages through autocrine TNF and ROS production. Blood 2012; 119: 2368-2375.

14. Schaer CA, Deuel JW, Bittermann AG, Rubio IG, Schoedon G, Spahn DR et al. Mechanisms of haptoglobin protection against hemoglobin peroxidation triggered endothelial damage. Cell Death Differ 2013; 20: 1569-1579.

15. Pimenova T, Pereira CP, Gehrig P, Buehler PW, Schaer DJ, Zenobi R. Quantitative mass spectrometry defines an oxidative hotspot in hemoglobin that is specifically protected by haptoglobin. J Proteome Res 2010; 9: 4061-4070.

16. Vallelian F, Schaer CA, Kaempfer T, Gehrig P, Duerst E, Schoedon G et al. Glucocorticoid treatment skews human monocyte differentiation into a hemoglobin-clearance phenotype with enhanced heme-iron recycling and antioxidant capacity. Blood 2010; 116: 5347-5356.

17. Ghosh S, Adisa OA, Chappa P, Tan F, Jackson KA, Archer DR et al. Extracellular hemin crisis triggers acute chest syndrome in sickle mice. J Clin Invest 2013; 123: 4809-4820.

18. Ong SE, Blagoev B, Kratchmarova I, Kristensen DB, Steen H, Pandey A et al. Stable isotope labeling by amino acids in cell culture, SILAC, as a simple and accurate approach to expression proteomics. Mol Cell Proteomics 2002; 1: 376-386.

19. Grunberg-Etkovitz N, Greenbaum L, Grinblat B, Malik Z. Proteasomal degradation regulates expression of porphobilinogen deaminase (PBGD) mutants of acute intermittent porphyria Biochim Biophys Acta 2006; 1762: 819-827.

20. Sacchetta P, Santarone S, Battista P, Di Cola D. Isolation of two high-molecular-mass proteinases from human erythrocytes. Eur J Biochem 1990; 191: 275-280.

21. Hoffman L, Rechsteiner M. Nucleotidase activities of the $26 \mathrm{~S}$ proteasome and its regulatory complex. J Biol Chem 1996; 271: 32538-32545

22. Xiong X, Chong E, Skach WR. Evidence that endoplasmic reticulum (ER)-associated degradation of cystic fibrosis transmembrane conductance regulator is linked to retrograde translocation from the ER membrane. J Biol Chem 1999; 274: 2616-2624.

23. Oberdorf J, Carlson EJ, Skach WR. Uncoupling proteasome peptidase and ATPase activities results in cytosolic release of an ER polytopic protein. J Cell Sci 2006; 119(Pt 2): 303-313.

24. Johnston JA, Ward CL, Kopito RR. Aggresomes: a cellular response to misfolded proteins. J Cell Biol 1998; 143: 1883-1898.
25. Fineschi S, Reith W, Guerne PA, Dayer JM, Chizzolini C. Proteasome blockade exerts an antifibrotic activity by coordinately down-regulating type I collagen and tissue inhibitor of metalloproteinase-1 and up-regulating metalloproteinase-1 production in human dermal fibroblasts. FASEB J 2006; 20: 562-564.

26. Buehler PW, D'Agnillo F, Schaer DJ. Hemoglobin-based oxygen carriers: from mechanisms of toxicity and clearance to rational drug design. Trends Mol Med 2010; 16: 447-457.

27. Kriegenburg F, Poulsen EG, Koch A, Kruger E, Hartmann-Petersen R. Redox control of the ubiquitin-proteasome system: from molecular mechanisms to functional significance. Antioxid Redox Signal 2011; 15: 2265-2299.

28. Breusing N, Grune T. Regulation of proteasome-mediated protein degradation during oxidative stress and aging. Biol Chem 2008; 389: 203-209.

29. Kanayama HO, Tamura T, Ugai S, Kagawa S, Tanahashi N, Yoshimura T et al. Demonstration that a human 26S proteolytic complex consists of a proteasome and multiple associated protein components and hydrolyzes ATP and ubiquitin-ligated proteins by closely linked mechanisms. Eur J Biochem 1992; 206: 567-578.

30. Santoro AM, Lo Giudice MC, D'Urso A, Lauceri R, Purrello R, Milardi D. Cationic porphyrins are reversible proteasome inhibitors. J Am Chem Soc 2012; 134: 10451-10457.

31. Lipiski M, Deuel JW, Baek JH, Engelsberger WR, Buehler PW, Schaer DJ. Human hp1-1 and hp2-2 phenotype-specific haptoglobin therapeutics are both effective in vitro and in Guinea pigs to attenuate hemoglobin toxicity. Antioxid Redox Signal 2013; 19: 1619-1633.

32. Gallien S, Bourmaud A, Kim SY, Domon B. Technical considerations for large-scale parallel reaction monitoring analysis. J Proteomics 2014; 100: 147-159.

33. Collier GS, Pratt JM, De Wet CR, Tshabalala CF. Studies on haemin in dimethyl sulphoxide/ water mixtures. Biochem J 1979; 179: 281-289.

34. Schaer CA, Vallelian F, Imhof A, Schoedon G, Schaer DJ. Heme carrier protein (HCP-1) spatially interacts with the CD163 hemoglobin uptake pathway and is a target of inflammatory macrophage activation. J Leukoc Biol 2008; 83: 325-333.

(c) (i) (2)(2) This work is licensed under a Creative Commons Attribution-NonCommercial-ShareAlike 3.0 Unported License. The images or other third party material in this article are included in the article's Creative Commons license, unless indicated otherwise in the credit line; if the material is not included under the Creative Commons license, users will need to obtain permission from the license holder to reproduce the material. To view a copy of this license, visit http://creativecommons.org/licenses/by-nc-sa/3.0/

Supplementary Information accompanies this paper on Cell Death and Differentiation website (http://www.nature.com/cdd) 